\title{
Shape optimization of solid-air porous phononic crystal slabs with widest full 3D bandgap for in-plane acoustic waves
}

\author{
Luca D'Alessandro ${ }^{\mathrm{a}, \mathrm{d}}$, Bichoy Bahr ${ }^{\mathrm{a}, 1}$, Luca Daniel ${ }^{\mathrm{b}}$, Dana Weinstein ${ }^{\mathrm{c}}$, Raffaele Ardito ${ }^{\mathrm{d}, *}$ \\ ${ }^{a}$ Dept. of Electrical Engineering and Computer Science, Massachusetts Institute of Technology Cambridge (MA) \\ ${ }^{b}$ Research Laboratory of Electronics, Massachusetts Institute of Technology Cambridge (MA), USA \\ ${ }^{c}$ Dept. of Electric and Computer Engineering, Purdue University West Lafayette (IN), USA \\ ${ }^{d}$ Dept. of Civil and Environmental Engineering, Politecnico di Milano Milan, Italy
}

\begin{abstract}
The use of Phononic Crystals (PnCs) as smart materials in structures and microstructures is growing due to their tunable dynamical properties and to the wide range of possible applications. PnCs are periodic structures that exhibit elastic wave scattering for a certain band of frequencies (called bandgap), depending on the geometric and material properties of the fundamental unit cell of the crystal. PnCs slabs can be represented by plane-extruded structures composed of a single material with periodic perforations. Such a configuration is very interesting, especially in Micro Electro-Mechanical Systems industry, due to the easy fabrication procedure. A lot of topologies can be found in the literature for PnCs with square-symmetric unit cell that exhibit complete 2D bandgaps; however, due to the application demand, it is desirable to find the best topologies in order to guarantee full bandgaps referred to in-plane wave propagation in the complete 3D structure. In this work, by means of a novel and fast implementation of the Bidirectional Evolutionary Structural Optimization technique, shape optimization is conducted on the hole shape obtaining several topologies, also with non square-symmetric unit cell, endowed with complete 3D full bandgaps for in-plane waves. Model order reduction technique is adopted to reduce the computational time in the wave dispersion analysis. The 3D features of the $\mathrm{PnC}$ unit cell endowed with the widest full bandgap are then completely analysed, paying attention to engineering design issues.
\end{abstract}

Keywords: Solid-Air Phononic Crystals, MEMS Resonators, 3D full bandgap, Shape optimization, BESO algorithm

\section{Introduction}

PnCs are represented by periodic media that exhibit bandgaps, i.e. bounded frequency regions for which the propagation of elastic waves through the crystal is impeded. The central frequency and the size of the bandgap basically depend on the materials employed in PnCs and on the shape of the unit cell. The use of Phononic Crystals $(\mathrm{PnC})$ at the macro and micro scale, and specifically in the field of Micro Electro-Mechanical Systems (MEMS), is nowadays improving in number and in technology, mainly due to the $\mathrm{PnC}$ versatility and easy fabrication. The review papers [1], [2], [3], [4] and [5] contain several examples of $\mathrm{PnC}$ application, among which: acoustic isolation, noise suppression, vibration attenuation, acoustic waveguides, acoustic super-lenses, negative refraction, acoustic cloaking, energy harvesting. The successful use of PnCs is also due to their tunable mechanical properties, in the presence of prestress $[6,7]$ or non-linear mechanical behavior [8].

This work is focused on $\mathrm{PnC}$ application in plane structures such as plates and slabs for acoustic wave confinement. Although the presented results are general, particular attention is given to PnC application in released MEMS resonators [9] and unreleased MEMS resonators [10]. The former kind of resonator can highly benefit from the presence of PnCs with the objective of reducing the wave radiation in the external support. On the other hand, unreleased resonators have the prerogative to overcome many challenges faced by traditional MEMS resonators such as non-linearity, power handling, residual stresses and yield [11]. In those cases, perforated slabs, i.e. solid-air $\mathrm{PnCs}$, are the most feasible and interesting for micro-scale fabrication, in order to obtain acoustic resonanting

\footnotetext{
${ }^{*}$ Corresponding author

Email addresses: lucadale@mit.edu, luca.dalessandro@polimi.it (Luca D’Alessandro), bichoy@ti.com (Bichoy Bahr), luca@mit.edu (Luca Daniel), danaw@purdue.edu (Dana Weinstein), raffaele.ardito@polimi.it (Raffaele Ardito)

${ }^{1}$ Present address: Kilby Labs, Texas Instruments Santa Clara (CA).
} 
cavities with frequency selective confinement. The typical manufacturing techniques at the micro-scale rely on lithographic processes. An example is represented by gallium nitride (GaN) resonators [10], that are based on GaN-on-Si heterostructures, comprised of $\operatorname{AlGaN}(25 \mathrm{~nm}) / \mathrm{GaN}(1.7 \mu \mathrm{m})$ grown on (111)-Si using Molecular Beam Epitaxy (MBE). The solid-air PnC is obtained by means of a deep $\mathrm{Cl}_{2}$ etch of $\mathrm{GaN}$ layer and a final release step with $\mathrm{XeF}_{2}$ etch of the silicon substrate. Due to technology issues, the hole shape is tipically an extrusion of a simple planar geometry, most commonly in literature a circle. That case, depending on the radius of the circular hole and the thickness of the PnC slab, could present full bandgap for 3D in-plane waves less than 15\% (gap to mid gap ratio, see [12]) or could not present any bandgap. It is worth mentioning that more complex 3D periodic structures could be fabricated by means of additive manufacturing, as it is shown in [13] at the macro-scale; additive manufacturing can be used for obtaining $\mathrm{PnC}$ at the micro-scale as well, e.g. by means of two-photon lithography [14] or Robocasting technique [15].

For any application, it is highly desirable to optimize the shape of the hole in the PnC unit cell in order to obtain the largest full 3D bandgap, while complying with manufacturability constraints. This is beneficial for several reasons, mainly related to the efficiency and robustness of the resonator, characterized by a minimal footprint. The present work aims to achieve the optimal shape, in terms of widest phononic bandgap, of the solid-air PnCs and to provide a thorough examination of the achieved topology, focusing on application goals. The optimization problem is specifically restricted to unit cells that contain a hole in the form of a contractible space. This means that (i) multiple holes and (ii) isolated regions are excluded a priori. While (ii) is an obvious restriction for solid-air PnCs, condition (i) represents a limitation, which differentiates our work from previous proposals and is fully justified by manufacturability considerations. In spite of such a limitation, we show that the proposed optimization algorithm leads to full 3D bandgap, whose width is greater than other scenarios in the literature.

Several research groups have treated the topological optimization of PnCs unit cell for 2D models (i.e. plane strain), mostly with two material inclusions: the pioneering work [16] tackles the problem by means of a gradient based algorithm (method of moving asymptotes, MMA); the use of genetic algorithms has been proposed for solid-solid PnC in [17], [18] and [19]. Recently, genetic algorithms have been also used for topology optimization of solid-air PnCs, see [20], [21], [22] for the 2D case and [23] for the full 3D case. In the present work, we consider the application of Bidirectional Evolutionary Structural Optimization (BESO) that has been demostrated well suited for Photonic Crystals topology optimization [24], solid-solid PnCs topology optimization [25] and in sistematic 2D topology optimization of solid-air [26] PnCs. In those studies, it is shown how BESO algorithm can work well in bandgap optimization problems and can be faster than other algorithms in terms of number of iterations necessary to find the optimal topology.

In the present work, the BESO algorithm is presented for the first time directly in full 3D shape optimization, in a novel and fast engineering perspective. The method is based on sensitivity analysis for the acoustic wave frequency, which is obtained through a 3D Finite Element (FE) approach that has been developed in a Matlab environment with a great attention to computational time issues. The shape optimization procedure starts from a simple hole extruded from a planar geometry (i.e. circle), for solid-air Gallium Nitride (GaN) PnC, and proceeds in order to evolve this geometry into the optimal one for maximum bandgap. The optimal shapes of the hole with respect to different bands of frequencies are found in less than 30 iterations, by adding and removing solid elements. The air domain is not taken into account in the model: in this way numerical instability issues due to interpolation between the two solid-air phases are not present and the number of unknowns for the FE solver is reduced. The efficiency of the proposed algorithm is further enhanced by the application of a model order reduction technique [27].

Several optimal topologies are presented related to different bandgaps, all of them with a single contractible hole in order to be easily feasible by micro-fabrication. In the second part of the paper, the dynamic behaviour of the optimal $\mathrm{PnC}$ (endowed with the widest bandgap) is analyzed with a 3D FE approach in order to understand the acoustic wave propagation phenomena. Comparison with 2D approximations and full 3D model are conducted: we specifically consider how the width of the bandgap varies at different thicknesses of the slab and what type of modes is responsible of the change of bandgap width. Finally, other materials which are typically adopted in MEMS industry are analyzed, such as Alluminium Nitride (AlN) and Silicon (Si).

\section{Finite element problem formulation}

PnC structures are periodic structures generally composed of two or more elastic material phases, solid or fluid. The theoretical framework is wave propagation in elastic inhomogeneous media, and it is governed by the 


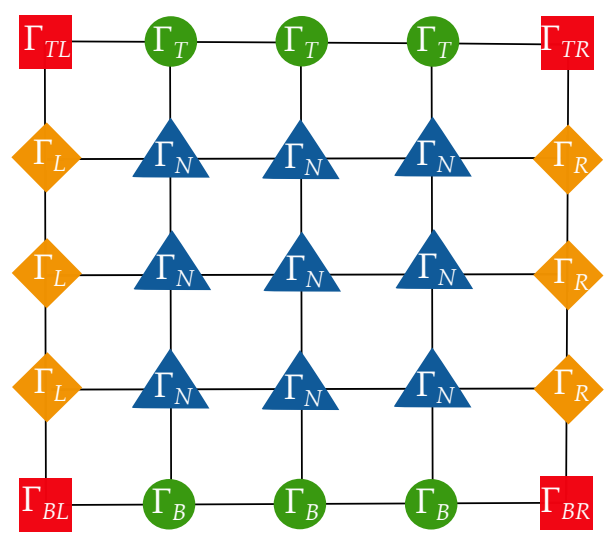

Figure 1: FE in-plane grid with nodes identified by type with respect to periodic boundary conditions.

Г: boundary, T: Top, B: Bottom, L: Left, R: Right, I: Internal.

The nodes identified by the same geometric figure (or color) are related by the bc.

(For interpretation of the references to color in this figure caption, the reader is referred to the web version of this paper.)

following elasto-dynamic equations [28]:

$$
\begin{array}{r}
\rho \frac{\partial^{2} u_{x}}{\partial t^{2}}=\frac{\partial}{\partial x}(\lambda \nabla \cdot \mathbf{u})+\nabla \cdot\left(\mu\left(\nabla u_{x}+\frac{\partial \mathbf{u}}{\partial x}\right)\right) \\
\rho \frac{\partial^{2} u_{y}}{\partial t^{2}}=\frac{\partial}{\partial y}(\lambda \nabla \cdot \mathbf{u})+\nabla \cdot\left(\mu\left(\nabla u_{y}+\frac{\partial \mathbf{u}}{\partial y}\right)\right) \\
\rho \frac{\partial^{2} u_{z}}{\partial t^{2}}=\frac{\partial}{\partial z}(\lambda \nabla \cdot \mathbf{u})+\nabla \cdot\left(\mu\left(\nabla u_{z}+\frac{\partial \mathbf{u}}{\partial z}\right)\right)
\end{array}
$$

where $\rho$ is the material density, $\lambda$ and $\mu$ are Lame's coefficients, $\mathbf{u}=\left[u_{x}, u_{y}, u_{z}\right]$ is the displacement vector and $u_{x}, u_{y}, u_{z}$ its cartesian components.

The case study analyzed in this paper is referred to PnC slabs composed of solid-air phase materials, where the 3D structure is the result of an extrusion from a planar geometry. Therefore it is possible to assume that the material parameters $\rho, \lambda$ and $\mu$ depend only on the $x$ and $y$ (in-plane) coordinates: $\rho=\rho(x, y), \lambda=\lambda(x, y), \mu=\mu(x, y)$. Suitable boundary conditions must be defined in order to define the complete dynamic problem on the unit cell:

- free boundary condition at the interface between the solid and air domain;

- periodic boundary conditions in the external boundary by means of the Floquet-Bloch theorem [29] [30]:

$$
\mathbf{u}=\mathbf{u}(\mathbf{r}, \mathbf{k})=\tilde{\mathbf{u}}(\mathbf{r}) e^{i \mathbf{k} \cdot \mathbf{r}} e^{i \omega t}
$$

where $\tilde{\mathbf{u}}(\mathbf{r})$ is the Bloch displacement vector over the unit cell. More details on the formulations can be found in our previous paper [31].

Referring to the unit cell domain, the weak form of Eq. (1) is discretized with FE technique choosing the linear cubic element for 3D analysis. In order to implement easily the shape optimization algorithm the in-plane FE mesh is a regular grid over the unit cell: each element can be treated as a "pixel" that can be assigned to solid or air domain. The out of plane mesh is also a regular grid, with out of plane - in plane element ratio around 6 and never more than 10, depending on the slab thickness. An important aspect is that, in order to decrease the computational cost, the air domain is not considered in the FE discretization.

The FE discretization yields the governing equation for wave propagation:

$$
\mathbf{w}^{T}(\mathbf{k})\left(\mathbf{K}-\omega^{2} \mathbf{M}\right) \mathbf{w}(\mathbf{k})=0
$$

where $\mathbf{k}=\left[k_{x}, k_{y}, 0\right]$ is the wave vector: only in-plane wave propagation is considered, in view of the specific geometry of the PnC slabs, so the third component of the wave vector is null. For the remaining symbols, 
$\mathbf{r}=\left[r_{x}, r_{y}, r_{z}\right]$ is the position vector, $\mathbf{K}$ and $\mathbf{M}$ are the assembled global stiffness and mass matrices, $\mathbf{w}(\mathbf{k})$ is the global vector of unknowns containing in order the $x, y$ and $z$ components of each node displacement.

Due to the organization of the nodes of the mesh (as shown in Fig. 1), it is possible to define the periodic boundary conditions in a matrix form:

$$
\mathbf{w}(\mathbf{k})=\mathbf{T}(\mathbf{k}) \tilde{\mathbf{w}}
$$

where $\tilde{\mathbf{w}}$ collects the so called "independent" nodal displacements, i.e. the degrees of freedom of the nodes on $\Gamma_{L}$, $\Gamma_{B L}, \Gamma_{B}, \Gamma_{B R}$ and $\Gamma_{N}$. The rectangular matrix $\mathbf{T}(\mathbf{k})$ contains the periodic relation between the nodes; its entries are only zeros, ones and $e^{i \mathbf{k} \cdot \Delta \mathbf{r}}$ values, being $\Delta \mathbf{r}$ the relative position vector between the nodes involved in the periodicity condition (further details can be found in [32] and in our previous paper [31]).

By introducing Eq. (4) in Eq. (3), one finds:

$$
\tilde{\mathbf{w}}^{T} \mathbf{T}^{H}(\mathbf{k})\left(\mathbf{K}-\omega^{2} \mathbf{M}\right) \mathbf{T}(\mathbf{k}) \tilde{\mathbf{w}}=0
$$

where ${ }^{H}$ stands for Hermitian transpose.

The following problem matrices are Hermitian and depend on the wave vector $\mathbf{k}$ :

$$
\begin{aligned}
\tilde{\mathbf{K}}(\mathbf{k}) & =\mathbf{T}^{H}(\mathbf{k}) \mathbf{K} \mathbf{T}(\mathbf{k}) \\
\tilde{\mathbf{M}}(\mathbf{k}) & =\mathbf{T}^{H}(\mathbf{k}) \mathbf{M} \mathbf{T}(\mathbf{k})
\end{aligned}
$$

Introducing Eq. (6) in Eq. (5):

$$
\tilde{\mathbf{w}}^{T}\left(\tilde{\mathbf{K}}-\omega^{2} \tilde{\mathbf{M}}\right) \tilde{\mathbf{w}}=0
$$

Eq. (7) must be solved in order to find eigenvalues $\omega_{n}^{2}$, eigenvectors $\tilde{\mathbf{w}}_{n}(\mathbf{r})$ and the associated dispersion relation at varying $\mathbf{k}$. It is important to notice that, since the governing matrices are Hermitian, all the eigenvalues are real. The description of the dynamic behavior of the $\mathrm{PnC}$ for all the possible vectors in the plane can be obtained by choosing a limited number of vectors, discretizing the boundary of the first Irreducible Brillouin Zone (IBZ) [33]. In Fig. 2, the bold square in $\mathbf{k}$-space is the Brilluin zone. Due to the symmetries of the unit cell, it is possible to focus the attention on some particular regions of the $\mathbf{k}$-space called first IBZ, as illustrated in Fig. 2: for $x-y$ symmetry it is possible to reduce the $\mathbf{k}$-space to the colored square, for square symmetry to the colored triangle. Many authors (see e.g. [29, 32]) claim that from the engineering point of view the maximum and minimum points of the bandgap can be determined focusing the attention on the $\mathbf{k}$ vectors along the boundary of the first IBZ, following the paths indicated in Fig. 3.

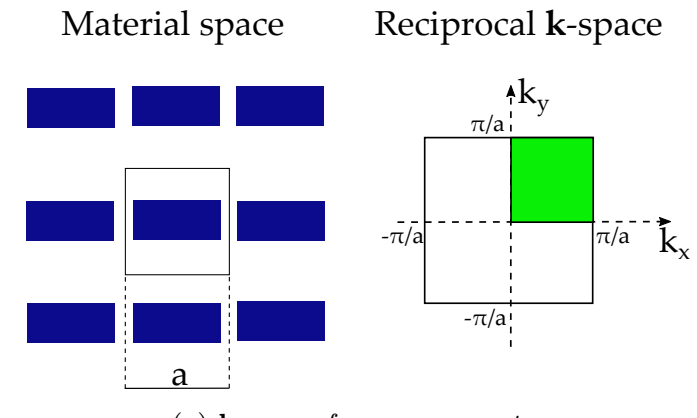

(a) $\mathbf{k}$-space for $x$-y symmetry.

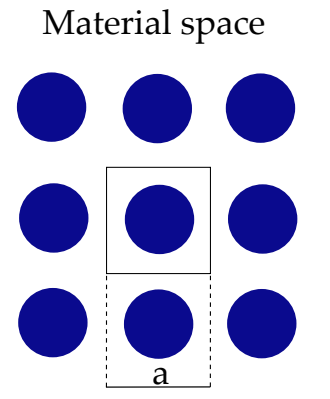

Reciprocal k-space

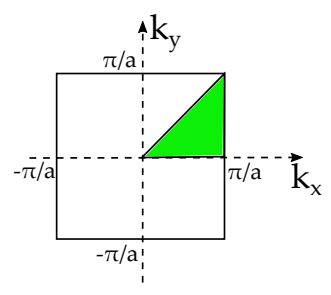

(b) $\mathbf{k}$-space for square symmetry.

Figure 2: Material and reciprocal space with the indication of the First Irreducible Brillouin Zone in the k-space.

One important point, which deserves a discussion here, is the possible inclusion of air in the FE model. The question does not seem to have a unique answer: for instance, in the case of application of genetic algorithms, the Authors in [20] and [21] do not include the air portion in the model (which is limited to plane strain); conversely, the Authors in [23], who consider a full 3D model, prefer to take into account the void sections of porous PnC by introducing some very compliant material, but they finally point out some numerical drawbacks of that choice. In the application of BESO algorithm, see [26], the introduction of air in the model seems unaivoidable, if one wants to implement the standard optimization procedure that relies on the material switch in some specific elements. Nevertheless, the robustness and the computational burden are highly affected by the fictitious modeling of the void sections. For this reasons, we prefer to consider a FE model which encompasses the solid part only, so that the dispersion diagram can be obtained in a shorter time without any numerical issue. Clearly, this choice entails some peculiar modification of the BESO optimization algorithm, which will be discussed in the next section. 


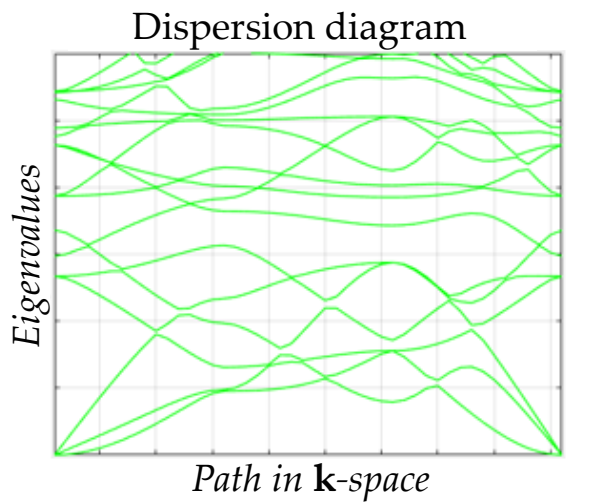

Path in k-space
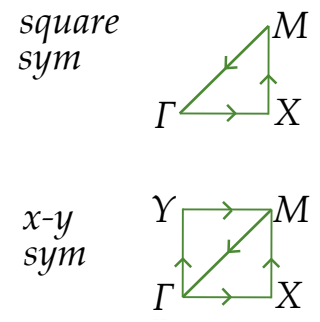

Figure 3: Dispersion diagram for k-vector along the boundary of the First Irreducible Brillouin Zone for different symmetries.

\section{Shape optimization problem formulation}

\subsection{Objective function}

The aim of this work is to find the shape of the single hole in solid-air PnCs that maximizes the bandgap between two adiacent eigenvalues.

The objective function of the optimization procedure is therefore the bandgap width normalized with respect to the central eigenvalue of the bandgap:

$$
\max _{\boldsymbol{\rho}}\left\{\frac{\Delta \omega^{2}}{\omega_{c e n}^{2}}=2 \frac{\min _{k} \omega_{n+1}^{2}(\mathbf{k}, \boldsymbol{\rho})-\max _{k} \omega_{n}^{2}(\mathbf{k}, \boldsymbol{\rho})}{\min _{k} \omega_{n+1}^{2}(\mathbf{k}, \boldsymbol{\rho})+\max _{k} \omega_{n}^{2}(\mathbf{k}, \boldsymbol{\rho})}\right\}
$$

where $\boldsymbol{\rho}$ is a vector that contains the material density of each element and $n$ is the number of the bottom eigenvalue that define the bandgap analyzed. The densities of the elements can only assume the values of 0 or 1, no interpolation between the two values is adopted: the element can be active and consequently it is taken into account in the FE calculations, or can be de-activated and consequently it is not taken into account in the calculations since it belongs to the air domain.

Some proper filters are introduced in the algorithm in order to avoid the creation of suspended regions of material and the so-called checkerboard effect, which is a common issues in optimization procedures [34]. As explained in the introduction, the algorithm is specifically studied in order to obtain holes in the form of a contractible space.

\subsection{Constraints}

A careful analysis must be conducted in order to define constraints for the shape optimization procedure, paying attention to micro-fabrication technology issues and to some specific troubles for solid-air PnC. The case of porous PnC may suffer some mesh dependency problems, which have been overlooked in some previous papers (e.g. [20]). Mesh dependency means that the finer the adopted FE mesh, the higher the bandgap, since the optimization algorithm tends to "erode" the material and to make as thin as possible the links between solid parts. That behaviour is connected to another important issue of porous $\mathrm{PnC}$, namely the structural worthiness, in terms of stiffness and/or resistance to external loads.

The former issue, i.e. mesh sensitivity, can be solved by applying cumbersome techniques, such as the BESO method with perimeter control [35]; in other cases, e.g. [21] and [22], a by far simpler solution has been found: the algorithm entail an additional constraint so that the geometrical width of each link between solid parts is larger than a certain threshold. That solution is effective to solve the mesh dependency, but, on the other hand, it does not ensure the satisfaction of stiffness limits and, most importantly, it may degrade the optimality of the bandgap. For instance, the constraint on each link impedes the creation of internal compliant parts, which do not alter the overall stiffness of the unit cell but may increase the bandgap by inducing some local resonance: two of the optimal shapes that we obtain, for higher modes, are endowed with that feature.

The introduction of explicit constraints on the stiffness parameters seems the ideal provision to avoid any trouble. Such an operation has been carried out in the literature by introducing complicate modifications of the optimization algorithm. The Authors in [23] propose the application of a multi-objective optimization process, with 
the achievement of Pareto frontiers between the two conflicting objectives of maximum bandgap and maximum stiffness. Conversely, the Authors in [26] introduce additional constraints for imposing that the stiffness parameters are larger than some pre-defined thresholds: the objective function is then modified via the Lagrange multiplier technique and the optimization algorithm is modified in order to account for the additional unknowns.

In the present paper, a simple, though effective, condition is introduced. The procedure of shape optimization gradually modifies the hole shape and can possibly end up near to the boundary of the unit cell: thus, we impose that a certain amount of lines of elements on the boundary square frame of the unit cell cannot be modified by the optimization algorithm. Therefore, the elements that belongs to this sort of "picture frame" are and remain in the solid domain throughout the optimization process (see Fig. 4). In that way, the mesh dependency issue is avoided in a global sense on the unit cell, without impeding the creation of internal weak links. Moreover, the bulk and shear stiffness parameters are always larger than the ones that are obtained for the configuration in Fig. 4: the number of lines that are not involved in the optimization process can be suitably tuned in order to fulfill some pre-defined requirements in terms of homogenized stiffness. A similar concept can be applied to overall strengths, which is dominated by local buckling in the case of membrane loads. The procedure is implemented in a straigthforward way and the optimization process maintains its outstanding performances in terms of convergence speed.

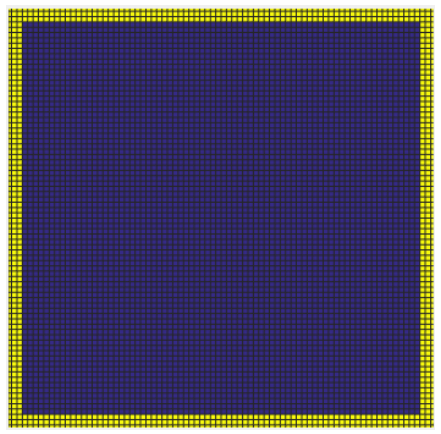

Figure 4: Square frame in the unit cell boundary (in-plane): elements in the outer region (light or yellow) are not involved in the shape optimization procedure in order to avoid mesh dependency and to impose some specific requirements on the structural worthiness.

\subsection{Sensitivity analysis}

The formulated optimization problem is a well suited candidate for the Evolutionary Structural Optimization (ESO) technique framework [36]. The eigenvalus, that define the objective function in Eq. (8), are given by:

$$
\omega_{n}^{2}=\frac{k_{n}}{m_{n}}, \quad k_{n}=\tilde{\mathbf{w}}_{n}^{T} \tilde{\mathbf{K}} \tilde{\mathbf{w}}_{n}, \quad m_{n}=\tilde{\mathbf{w}}_{n}^{T} \tilde{\mathbf{M}} \tilde{\mathbf{w}}_{n}
$$

where $k_{n}$ is the modal stiffness, $m_{n}$ is the modal mass and $\mathbf{w}_{n}=\mathbf{T} \tilde{\mathbf{w}}_{n}$ is the eigenvector.

The main assumption is that modifying the density of only few elements the shape of the eigenvector will not change significantly. The assumption that the mode shape does not change significantly in between design cycles has been commonly used in frequency optimization [37]:

$$
\delta\left(\tilde{\mathbf{w}}_{n}\right) \approx 0
$$

It is worth underlining that, according to the original formulation of ESO algorithm for solid-air problems, the calculation of sensitivity is always referred to the removal of an element from the present configuration. By removing one single element $e$, the eigenvalue will change as follows:

$$
\delta\left(\omega_{n}^{2}\right)=\frac{1}{m_{n}} \delta k_{n}-\frac{k_{n}}{m_{n}^{2}} \delta m_{n}
$$

where the variation of $k_{n}$ and $m_{n}$ can be easily obtained by extrapolating the entries referred to element $e$ from the eigenvectors and from the governing matrices:

$$
\delta k_{n}=\tilde{\mathbf{w}}_{n}^{T} \delta \tilde{\mathbf{K}} \tilde{\mathbf{w}}_{n}=-\tilde{\mathbf{w}}_{n}^{e T} \tilde{\mathbf{K}}^{e} \tilde{\mathbf{w}}_{n}^{e}
$$




$$
\delta m_{n}=\tilde{\mathbf{w}}_{n}^{T} \delta \tilde{\mathbf{M}} \tilde{\mathbf{w}}_{n}=-\tilde{\mathbf{w}}_{n}^{e T} \tilde{\mathbf{M}}^{e} \tilde{\mathbf{w}}_{n}^{e}
$$

The sensitivity parameter $\alpha_{n}^{e}$ expresses how the eigenvalue increases by removing one single element:

$$
\alpha_{n}^{e}=\delta\left(\omega_{n}^{2}\right)=\frac{1}{m_{n}} \tilde{\mathbf{w}}_{n}^{e T}\left(\omega_{n}^{2} \tilde{\mathbf{M}}^{e}-\tilde{\mathbf{K}}^{e}\right) \tilde{\mathbf{w}}_{n}^{e}
$$

The sensitivity of the bandgap width is therefore calculated as follows:

$$
\alpha_{b g(n, n+1)}^{e}=\alpha_{n+1}^{e}-\alpha_{n}^{e}
$$

Referring to Eq. (15), it is possible to implement two strategies in order to increase the bandgap width, namely:

(a) $\rho_{e}=0$ for element $e$ that presents $\alpha_{b g(n, n+1)}^{e}>0$;

(b) $\rho_{e}=1$ for element $e$ that presents $\alpha_{b g(n, n+1)}^{e}<0$.

The most obvious procedure is represented by strategy (a) (typical of the standard ESO), since the possibility (b) is actually trivial due to the fact that it suggests to activate elements that are already active.

The novelty introduced below defines the shape optimization procedure of the air hole:

(A) $\rho_{e}=0$ for element $e$ nearby the hole that presents $\alpha_{b g(n, n+1)}^{e}>0$;

(B) $\rho_{E}=1$ for element $E$ not yet active -in the hole- that presents $\alpha_{b g(n, n+1)}^{n e a r e}<0$.

The proposed algorithm, which includes both conditions (A) and (B), is bidirectional since new elements can be activated in the hole. It is worth noting that the topological condition near e is introduced, because the air domain is not modelled in our case and, as a consequence, there is no sensitivity connected to such elements. The elements in the hole are activated if the sensitivity condition is fulfilled on the closest element on the hole's edge.

The optimization procedure in 3D must face the fact that the fabrication technology is such that the hole must be an extrusion of a 2D shape: in general, it is not possible to have modifications of the hole shape along the hole thickness. It is hence necessary to define a procedure that takes into account the different layers of elements in the thickness and the optimization algorithm must remove or add all elements column by column. Consequently, two alternatives arise: (i) a proper combination of the $\alpha_{b g(n, n+1)}$ values in the different planes along the same column is considered (e.g. the mean values along the thickness); (ii) reference is made to one particular layer of element. Since the basic difference with respect to a $2 \mathrm{D}$ optimization procedure (focused on in-plane and/or out-of-plane modes) is the presence of flexural and torsional modes that propagates as in-plane waves, it is necessary to choose a strategy that takes into account the $\alpha_{b g(n, n+1)}$ for those 3D modes in the sensitivity analysis. By considering the mechanical behavior of thin slabs subject to bending or torsion, one easily realizes that the upper or lower skins of the unit cell represent a good choice. In fact, 2D modes do not vary in the thickness and therefore choosing one specific plane for the sensitivity extraction does not alterate the final outcome; moreover, torsional and flexural modes present significant displacement magnitudes far from the neutral axis (top/bottom of the unit cell). We choose the upper skin as the reference plane, so from now on $\alpha_{b g(n, n+1)}$ values are referred to this plane.

\subsection{Shape optimization algorithm}

Following the path of reasoning introduced in the previous section, the core of the algorithm is:

$$
\begin{array}{lll}
\rho_{e}=0 & \text { for } & e_{(\text {nearby hole })} \mid \alpha_{b g(n, n+1)}^{e}>0 \\
\rho_{e}=1 & \text { for } & E_{(\text {nearby solid }- \text { in the hole })} \mid \alpha_{b g(n, n+1)}^{\text {near e }}<0
\end{array}
$$

Eq. (16a) defines the elements to be de-activated and Eq. (16b) defines the elements to be activated at each iteration.

The value $n$ is chosen at the beginning of the optimization procedure corresponding to the bottom eigenvalue of the bandgap that will be optimized. The constraint is that the procedure can not involve the element in the first $b^{*}$ lines of the border of the unit cell. The optimization scheme is described in Fig. 5.

The parameters governing each BESO iteration (see Fig. 5) are:

- nlines $^{d}$ max number of lines of solid-elements near to the hole to be de-activated; 


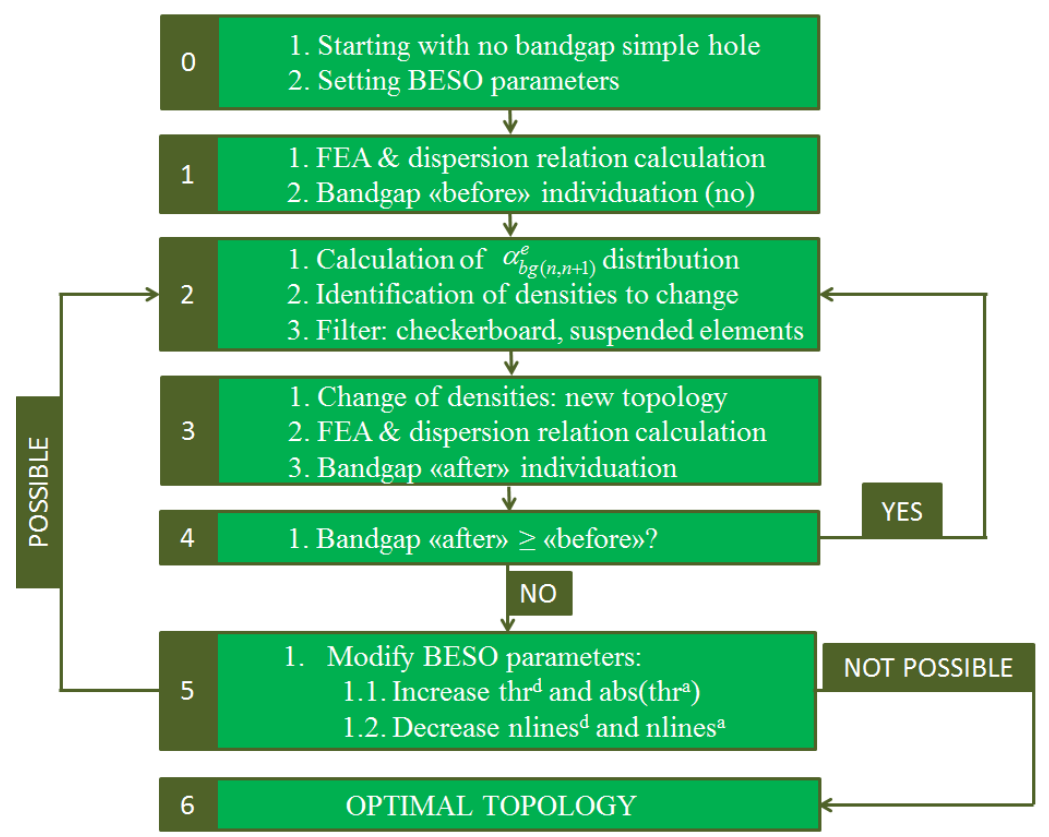

Figure 5: Block scheme of the optimization algorithm.

- nlines $^{a}$ max number of lines of hole-elements near to the solid to be activated;

- nelem $^{d}$ max number of elements in nlines $^{d}$ to be de-activated;

- nemel $^{a}$ max number of elements in nlines $^{a}$ to be activated;

- $t h r^{d}$ threshold for elements to be de-activated, $0<t h r^{d}<1$;

- $t h r^{a}$ for elements to be activated, $-1<t h r^{a}<0$.

The absolute values of the thresholds are limited to unity because the sensitivity values are normalized: the sensitivities for elements to be de-activated are divided by the maximum value attained in the specific set; conversely, for the elements that can be added, the normalization is carried out with respect to the minimum value, keeping the original sign. The normalized sensitivity are denoted by $\bar{\alpha}_{b g(n, n+1)}^{e}$. At each iteration, elements to be de-activated are:

$$
\mathbf{e}^{d}=\left\{e \quad \mid \quad \bar{\alpha}_{b g(n, n+1)}^{e}>t h r^{d}, \quad e \in \text { nlines }^{d}\right\}
$$

At each iteration, elements to be activated are:

$$
\mathbf{e}^{a}=\left\{E \quad \mid \text { E near } e, \quad e \quad \mid \bar{\alpha}_{b g(n, n+1)}^{e}<t_{h r}^{a}, \quad E \in \Gamma_{\text {hole }}, \quad E \in \text { nlines }^{a}\right\}
$$

where $\Gamma_{\text {hole }}$ identifies the boundary of the solid domain near by the air domain (i.e. hole). The two alternatives at point 5 in Fig. 5 are referred to the fact that the absolute value of the thresholds can not be larger than or equal to unity and that the number of lines should be larger than zero.

\section{Model order reduction}

The application of model order reduction is extremely advantageous in optimization problems: the first application can be found in [38], that studied the structural optimization of a micro-accelerometer including transient dynamic effects. In the present case, a model order reduction technique is adopted in order to decrease the computational time in the calculation of the wave dispersion diagram. The problem to be solved per each iteration of the optimization procedure is composed of several eigenvalue problems (one per each $\mathbf{k}$-vector considered in the first IBZ): it is possible to solve the problem by referring only to a few $\mathbf{k}$-vectors, called basis of the model order reduction technique, namely the ones corresponding to the symmetry points of the lattice [27] (see Fig. 6). The $m$ eigenvectors computed by solving Eq. 7 for the chosen $\mathbf{k}$-basis are collected in the $\boldsymbol{\Psi}$ matrix, which at the end of the procedure has dimensions $n \times m$ with $m \ll n$. 


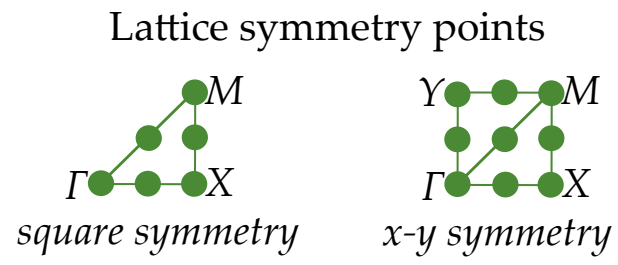

Figure 6: The dots represents the lattice symmetry points in $\boldsymbol{k}$-space chosen as basis for model order reduction.

The displacement vector $\tilde{\mathbf{w}}$ in Eq. 7 can be expressed in terms of the reduced basis as follows:

$$
\tilde{\mathbf{w}}=\boldsymbol{\Psi} \tilde{\mathbf{v}}
$$

where $\tilde{\mathbf{v}}$ is the reduced eigenvector with dimensions $m \times 1$. The eigenvalue problem for the $\mathbf{k}$-vectors in the symmetry points is rewritten as:

$$
\tilde{\mathbf{v}}^{T}\left(\tilde{\tilde{\mathbf{K}}}-\omega^{2} \tilde{\tilde{\mathbf{M}}}\right) \tilde{\mathbf{v}}=0
$$

where $\tilde{\tilde{\mathbf{K}}}=\boldsymbol{\Psi}^{T} \tilde{\mathbf{K}} \boldsymbol{\Psi}$ and $\tilde{\tilde{\mathbf{M}}}=\boldsymbol{\Psi}^{T} \tilde{\mathbf{M}} \boldsymbol{\Psi}$ are the new governing matrices which have the reduced dimensions $m \times m$. To recover the full solution one can use Eq. 19.

Fig. 7 contains the comparison between dispersion relations calculated with reduced order model and without the reduced order model: the two diagrams are almost undistinguished, thus confirming the validity of the proposed approach. Similar results are obtained also for the final shape, after the completion of the optimization procedure.

The model order reduction is crucial for tackling fully 3D problems, which are large scale problems by their very nature. In terms of computational time: to solve $31 \mathrm{FE}$ eigenvalue problems (one per each $\mathbf{k}$-vector) with around 138000 unknowns each, a computer characterized by 2 processors $2.40 \mathrm{GHz}$ each and $64 \mathrm{~Gb}$ RAM, without parallel computing, takes approximately 20 min for reduced order model solution and 130 min for full model solution. The huge computational saving is essential for keeping a reasonable overall execution time. 

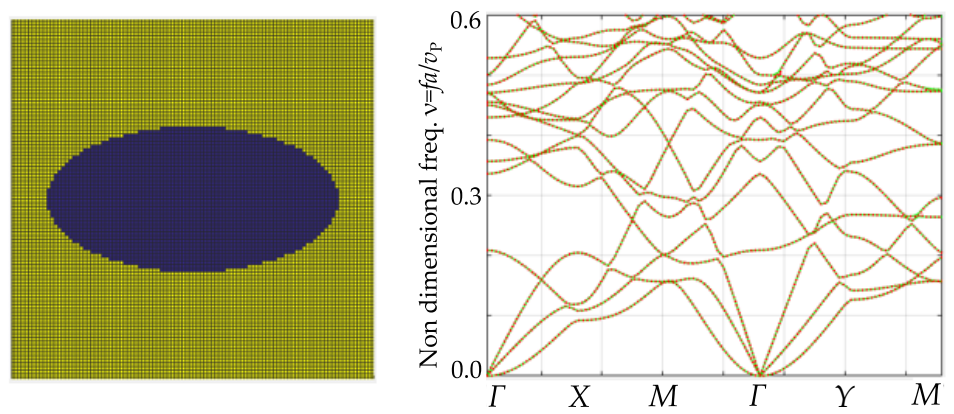

(a) Dispersion diagrams obtained by means of reduced $3 D$ model (solid green line) and full $3 D$ model (dashed red line), $x-y$ symmetry, ellipsoidal hole's view from the top.
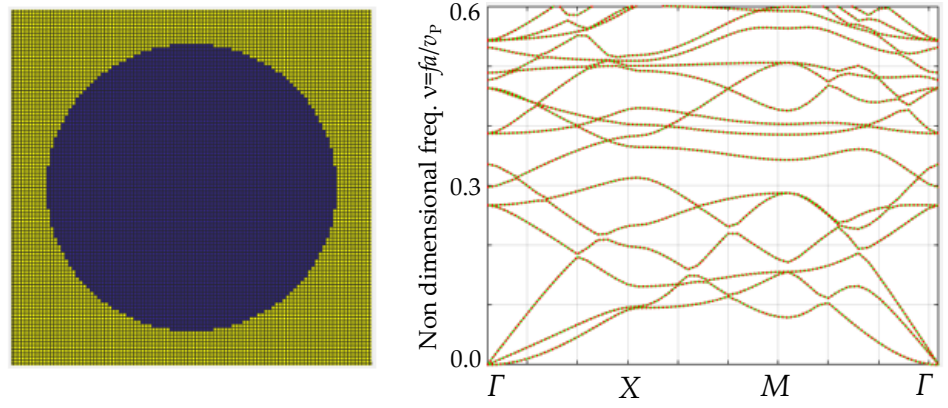

(b) Dispersion diagrams obtained by means of reduced 3D model (solid green line) and full $3 D$ model (dashed red line), square symmetry, circular hole's view from the top.

Figure 7: Comparison between reduced 3D model (solid green line) and full 3D model (dashed red line) for both $x$ - $y$ symmetry and square symmetry. 


\section{Numerical examples for shape optimization}

In this Section, several optimization results are presented, corrisponding to different bandgaps. The procedure starts from a circular hole, of radius $R=0.4 a$, in a parallalepiped volume with square base of dimension $a$ and thickness $a / 2$; the starting topology is not endowed with a full 3D bandgap as shown in Fig. 8. It is worth reminding that, from this point on, the dispersion diagrams are given in terms of non-dimensional frequency, i.e. the frequency times the unit cell size divided by the wave propagation velocity. This representation is useful in order to execute some comparison with previous results. Accordingly, the relative bandgap width will be referred to frequency, instead of eigenvalues. After few interations, always less than 30, the shape optimization procedure ends with the optimized hole shape with respect to the band of frequencies chosen.

The material considered in these analyses is $\mathrm{GaN}$, which is transversely isotropic, with isotropic properties in the $x-y$ plane (for material properties, please refer to Tab. 1).
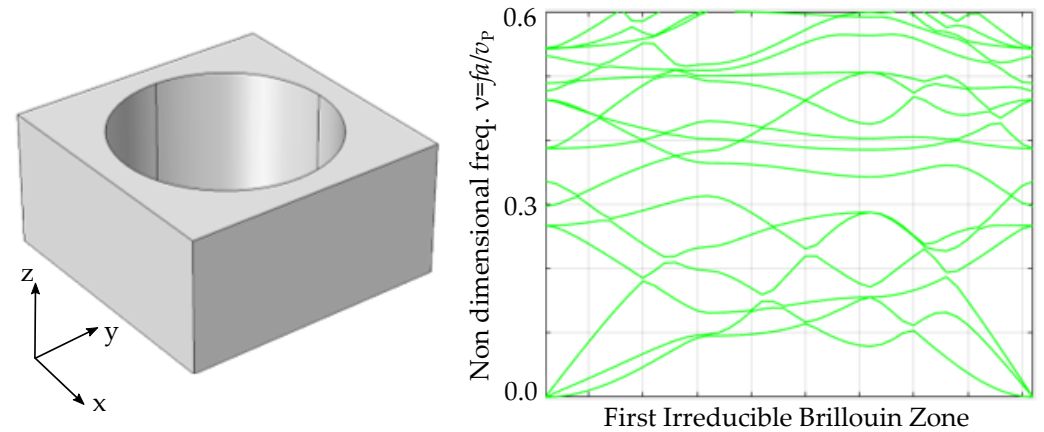

Figure 8: Starting topology with corresponding dispersion relation: no full bandgap. $f=\omega_{n} / 2 \pi$ is the frequency, $a$ is the square unit cell dimension, $v_{P}=\sqrt{\frac{\lambda+2 \mu}{\rho}}$

Table 1: Materials parameters for 3D analyses. $C_{i j}$ is the stiffness or elasticity matrix.

\begin{tabular}{cccccccc}
\hline Material & $C_{11}$ & $C_{12}$ & $C_{13}$ & $C_{33}$ & $C_{44}$ & $C_{66}$ & $\rho$ \\
& {$[G P a]$} & {$[G P a]$} & {$[G P a]$} & {$[G P a]$} & {$[G P a]$} & {$[G P a]$} & {$\left[\mathrm{kg} / \mathrm{m}^{3}\right]$} \\
\hline $\mathrm{GaN}$ & 390.00 & 145.00 & 106.00 & 398.00 & 105.00 & 122.50 & 6150 \\
$\mathrm{AlN}$ & 396.00 & 132.00 & 132.00 & 396.00 & 132.00 & 132.00 & 3255 \\
$\mathrm{Si}$ & 217.33 & 84.52 & 84.52 & 217.33 & 66.41 & 66.41 & 2329 \\
\hline
\end{tabular}

In Fig. 9 the optimal shapes for different chosen badgaps are presented. The optimization algorithm is applied sistematically in order to obtain the optimal bandgap for increasing eigenvalue number. It is worth noting that no bandgap can be found below the $6^{\text {th }}$ eigenvalue, thus confirming the results presented in [23]. The widest relative bandgap, for the specific $t / a$ ratio, is equal to $64 \%$ and corresponds to the lowest eigenmodes. A similar value is obtained between the $12^{\text {th }}$ and $13^{\text {th }}$ eigenvalues: that case corresponds to a similar topology, with a different disposition of the links between the solid portions. When examining the higher eigenmodes, e.g. Fig. 9d, one finds the appareance of internal resonating portions, connected to the main frame by means of weak links: such an optimal shape could not be obtained if the overall constraint in [21] had been implemented. Same thing for the shape in Fig. 9f: in that specific case, the optimal shape is obtained by introducing the $x-y$ symmetry condition. 

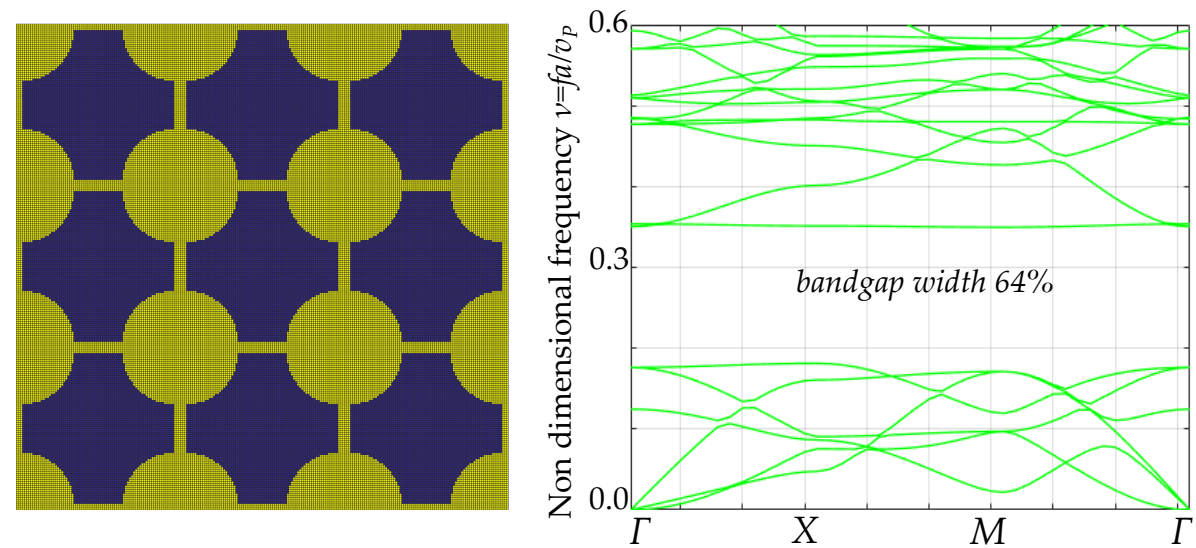

(a) Unit cell and full bandgap (64\%) between $6^{\text {th }}$ and th $^{\text {th }}$ eigenvalues.
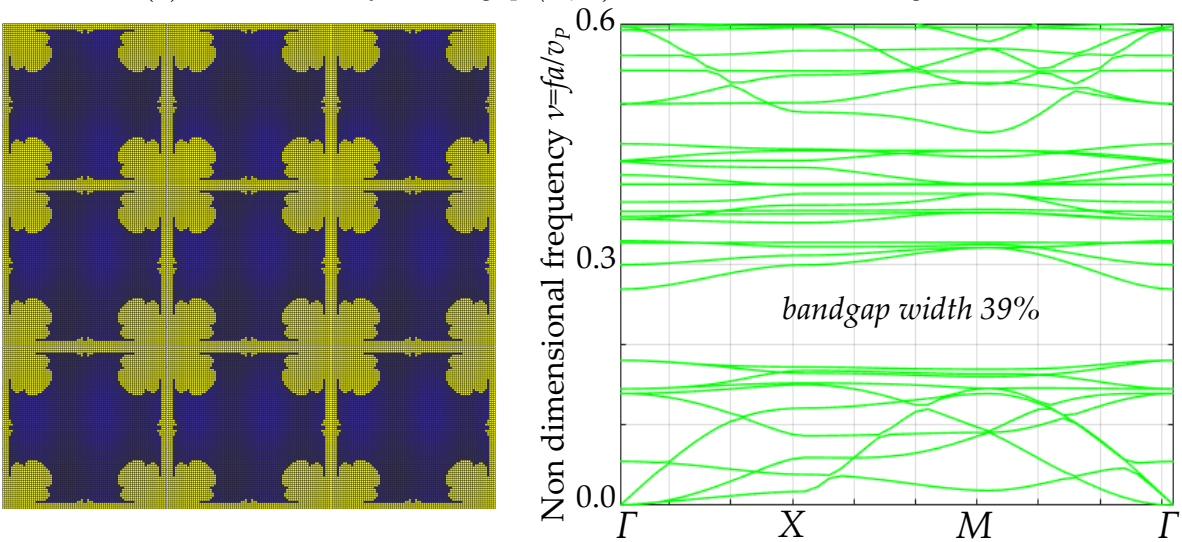

(b) Unit cell and full bandgap (39\%) between $10^{\text {th }}$ and $11^{\text {th }}$ eigenvalues.
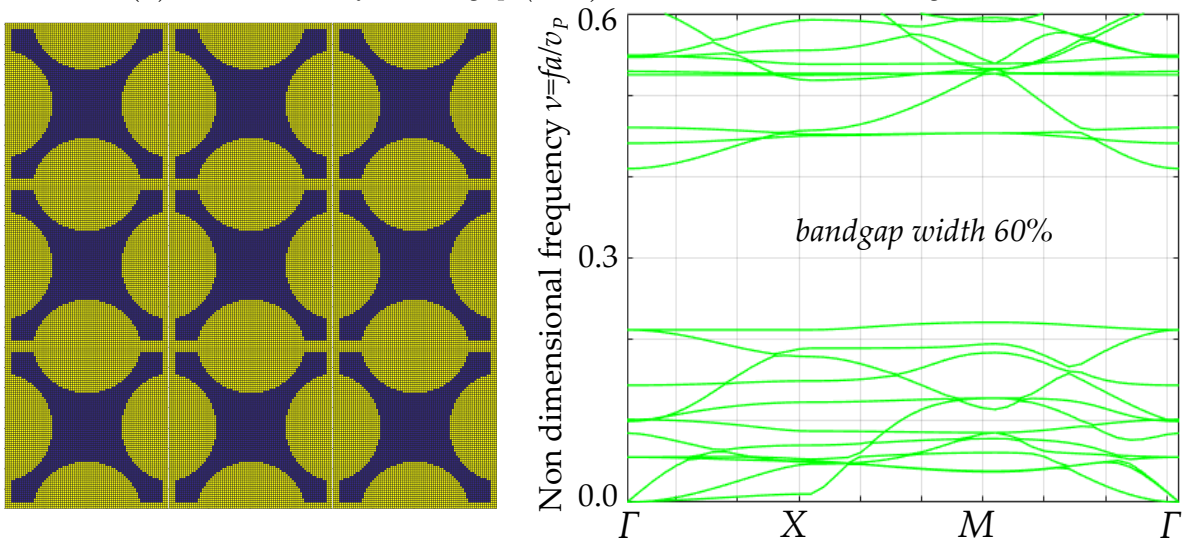

(c) Unit cell and full bandgap (60\%) between $12^{\text {th }}$ and $13^{\text {th }}$ eigenvalues.

Figure 9: Unit cells with corresponding hole topologies and associated 3D full bandgaps for in-plane waves propagation at different eigenvalues (cont.). 

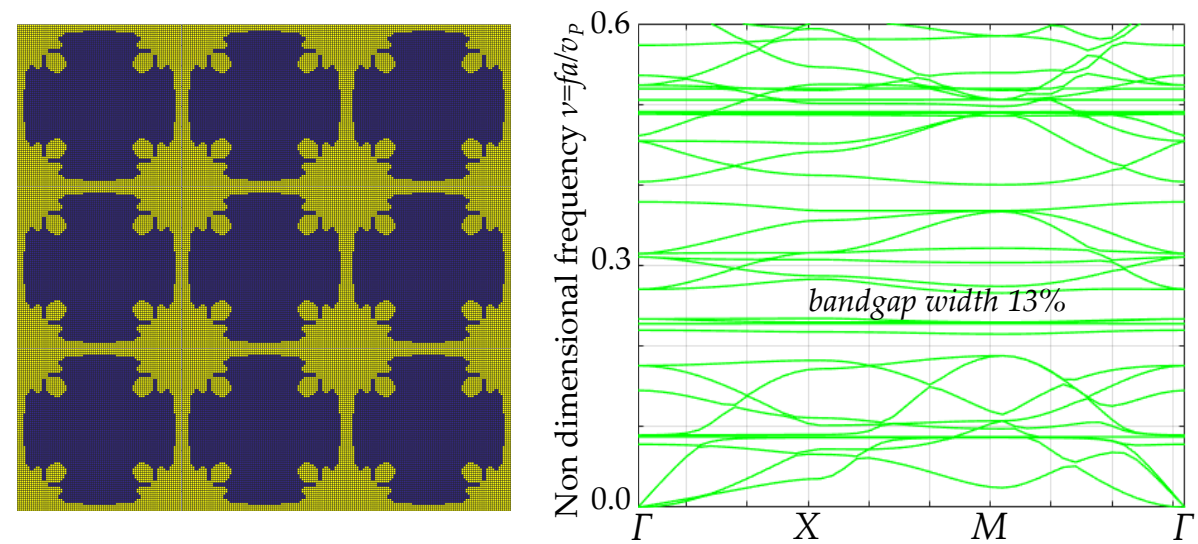

(d) Unit cell and full bandgap (13\%) between $14^{\text {th }}$ and $15^{\text {th }}$ eigenvalues.
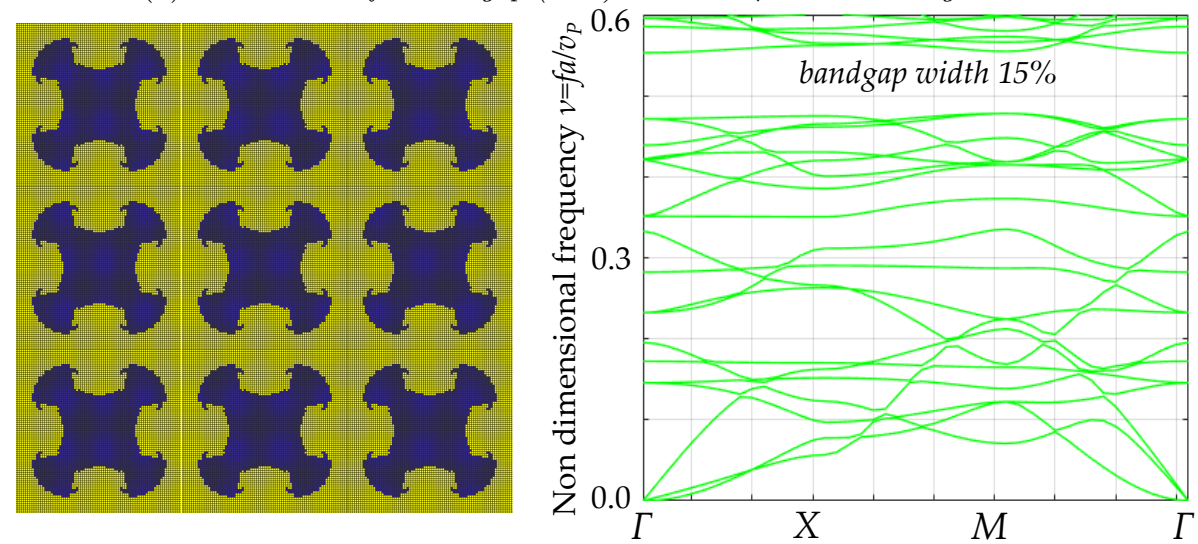

(e) Unit cell and full bandgap (15\%) between $19^{\text {th }}$ and $20^{\text {th }}$ eigenvalues.
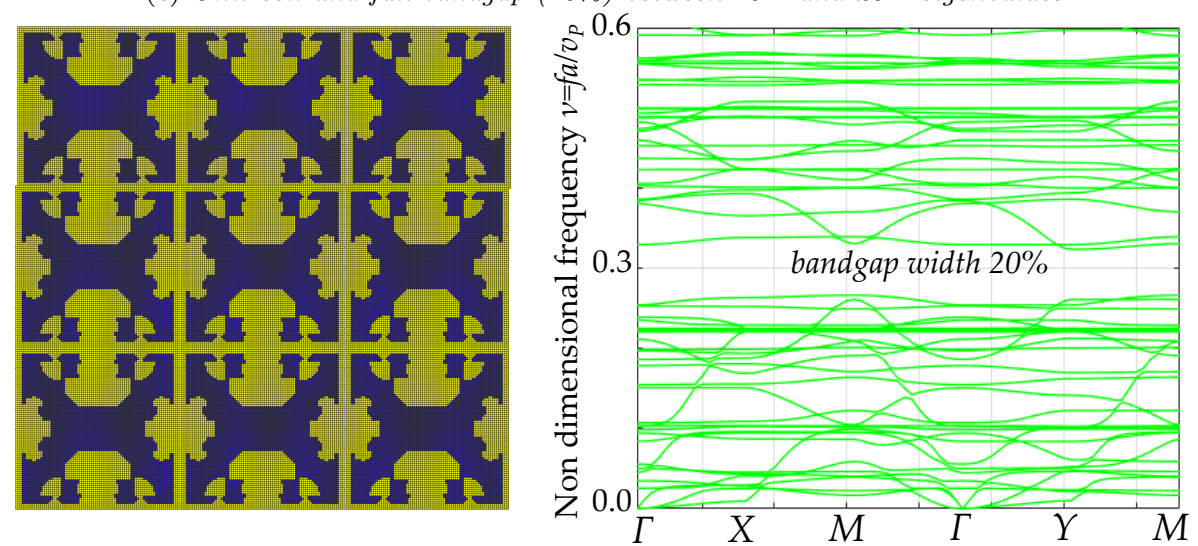

(f) Unit cell and full bandgap (20\%) between $28^{\text {th }}$ and $29^{\text {th }}$ eigenvalues.

Figure 9: Unit cells with corresponding hole topologies and associated 3D full bandgaps for in-plane waves propagation at different eigenvalues. 


\section{Analysis of the widest-bandgap unit cell}

The unit cell of Fig. 9a presents the widest full bandgap for 3D in-plane wave propagation. That configuration is now examined with reference to several aspects. First, we consider the comparison of 3D analysis with 2D approximations, in order to understand which one may fit better in this kind of problem. Then, it is necessary to analiyze how the bandgap width varies with respect to the thickness of the slab (it must be noticed that the optimal shape found in the previous section is referred to a particular thickness). Finally, attention will be focussed on the basic features of the of modal shapes that correspond to the eigenvalues that define the bandgap.

\subsection{Comparison with $2 D$ approximations.}

In this section the results of $3 \mathrm{D}$ analyses for PnC slabs are compared with the $2 \mathrm{D}$ approximations (plane strain and plane stress), that are commonly used in the literature for this kind of problems.

The comparison is conducted for four ratios $t / a$ (where $t$ is the slab thickness and $a$ is the in plane dimension of the unit cell), that identify the typical range of the MEMS resonators' applications (see Fig. 10). The results of $2 \mathrm{D}$ analyses, which are plotted in red dotted lines, are obviously the same for all the $t / a$ ratios. At a first glance, one can easily realize the importance of 3D analyses, since many different eigenmodes show up when this kind of model is considered. In all the cases, the 3D eigenmodes have a strong effect on the bandgap: for $t / a=0.0417$ the bandgap is completely destroyed, whereas for the other cases a bandgap still exists, but its width is greatly reduced. Clearly, the fully 3D analyses encompass also the 2D modes: by comparing the dispersion diagrams for the red dotted lines and the green line, one finds that, for the range of thicknesses analysed, the plane stress approximation presents the best fit to $3 \mathrm{D}$ results.

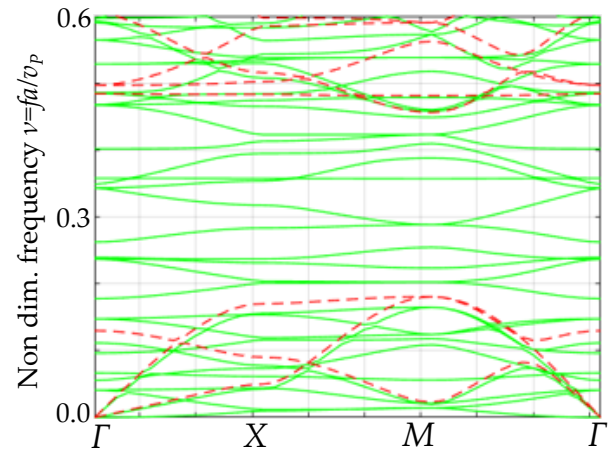

(a) Comparison between $2 D$ plane strain (red dashed) and $3 D$ (green full) models for $t / a=0.0417$.

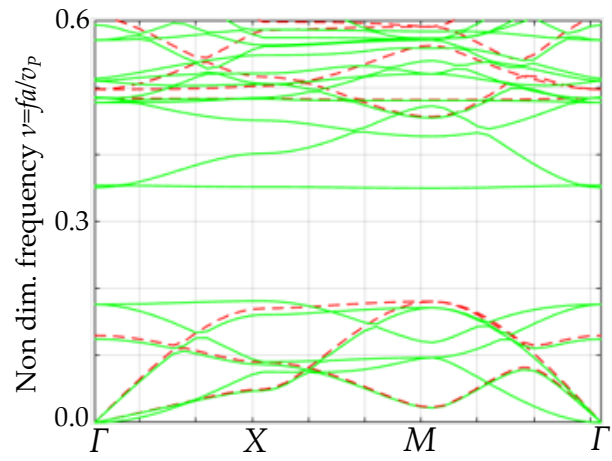

(c) Comparison between $2 D$ plane strain (red dashed) and $3 D$ (green full) models for $t / a=0.5000$.

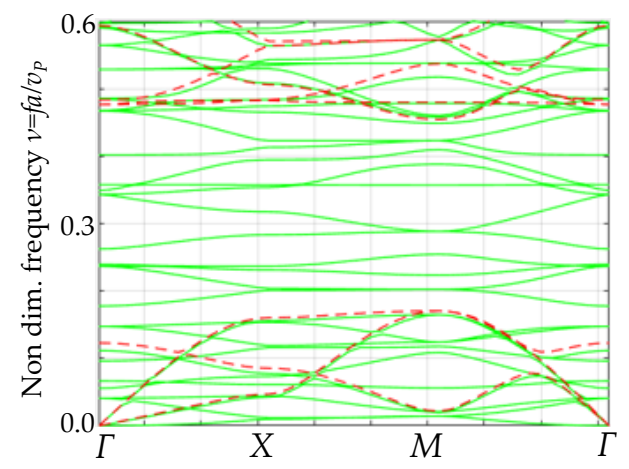

(b) Comparison between 2D plane stress (red dashed) and $3 D$ (green full) models for $t / a=0.0417$.

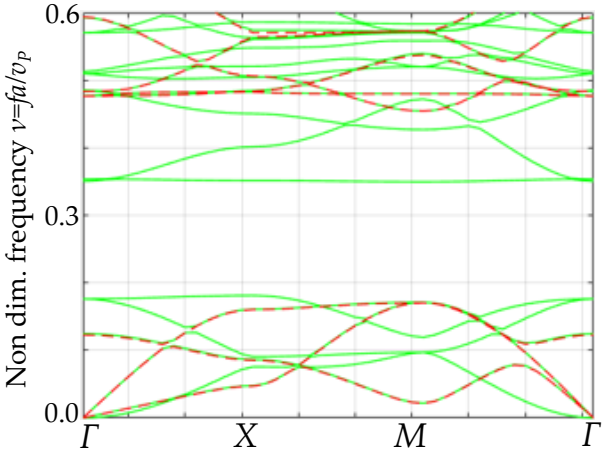

(d) Comparison between $2 D$ plane stress (red dashed) and $3 D$ (green full) models for $t / a=0.5000$.

Figure 10: Comparison between 2D plane strain (red dashed) and 3D (gren full) models for different t/a values (cont.). 


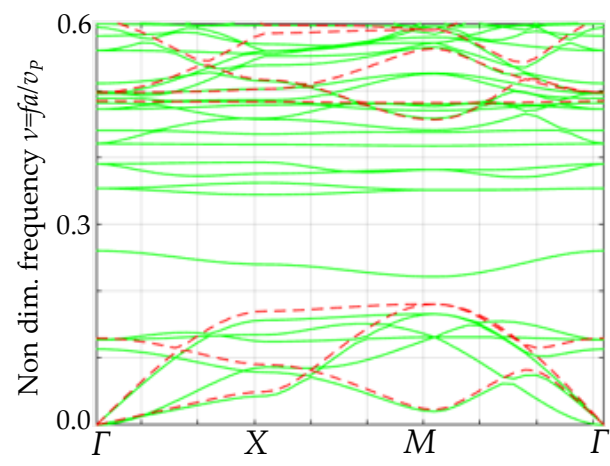

(e) Comparison between $2 D$ plane strain (red dashed) and $3 D$ (green full) models for $t / a=1.0000$.

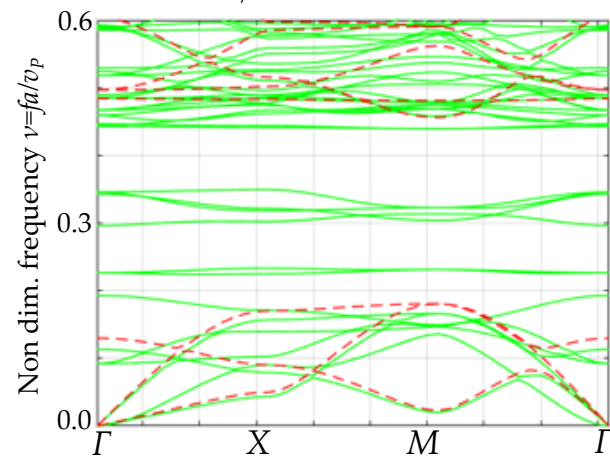

(g) Comparison between $2 D$ plane strain (red dashed) and $3 D$ (green full) models for $t / a=1.5000$

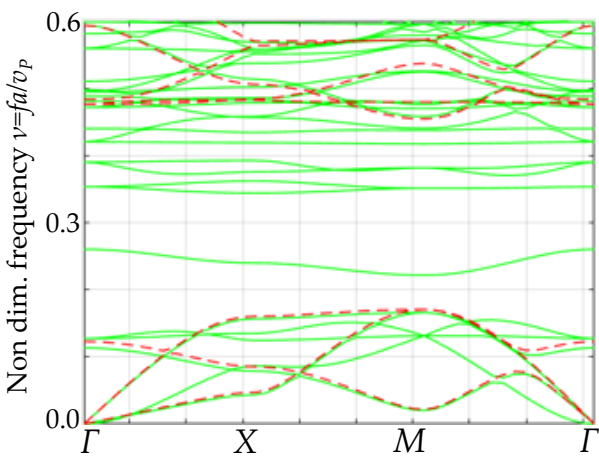

(f) Comparison between $2 D$ plane stress (red dashed) and $3 D$ (green full) models for $t / a=1.0000$.

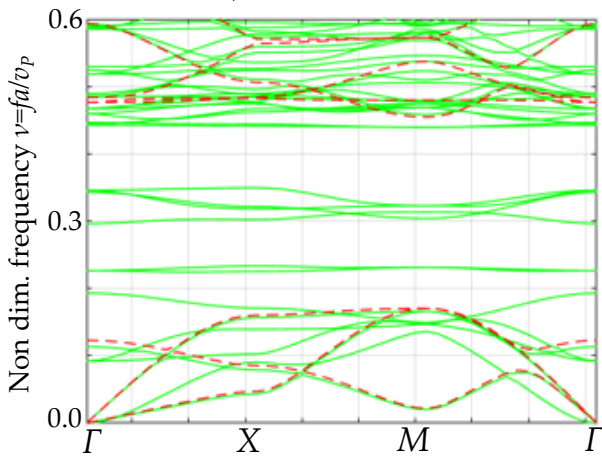

(h) Comparison between $2 D$ plane stress (red dashed) and $3 D$ (green full) models for $t / a=1.5000$.

Figure 10: Comparison between 2D plane strain (red dashed) and 3D (gren full) models for different $t / a$ values.

\subsection{Bandgap at varying thickness}

The examination of Fig. 10 shows that 3D bandgap for in-plane waves is highly influenced by the ratio $t / a$. Therefore, we carried out a set of analyses in order to understand how the bandgap width changes at varying thickness: the results are summarized in Fig. 11, that contains the plots of the $6^{\text {th }}$ and $7^{\text {th }}$ eigenfrequencies for different aspect ratios of the unit cell. The maximum bandgap width for the optimal shape is attained when $t / a=0.583$ : at that value, the relative bandgap width between $6^{\text {th }}$ and $7^{\text {th }}$ eigenvalues is equal to $76 \%$.

In Fig. 12 the dispersion relations at different thickness are shown in order to understand which modes are the main characters of the bandgap width variation. One finds that for low $t / a$ values no bandgap exists (Fig. 12a); then, the bandgap width gradually increases because a couple of modes start to move towards the high frequency region (Fig. 12b-d); in correspondence of the best aspect ratio, a different mode enters the bandgap (Fig. 12e) and then gradually moves towards the low frequency region (Fig. 12f-g). For higher thickness, different modes come into play. 


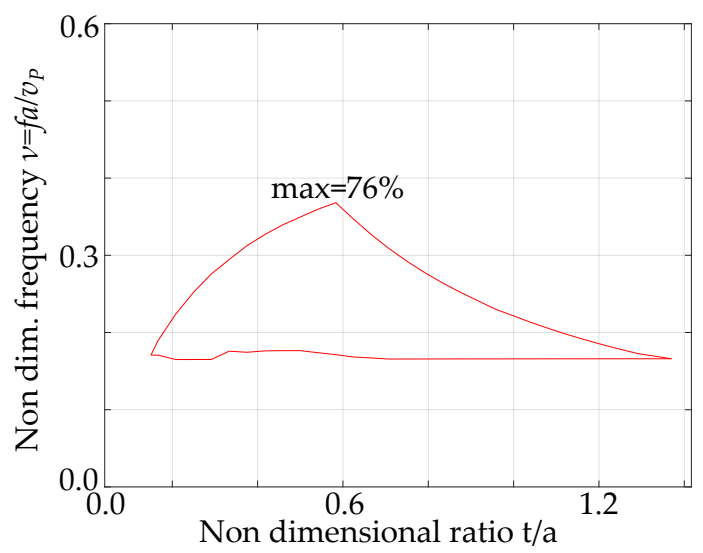

Figure 11: $6^{\text {th }}$ and $7^{\text {th }}$ bandgap width at different ratios between thickness $t$ and unit cell in-plane dimension a.

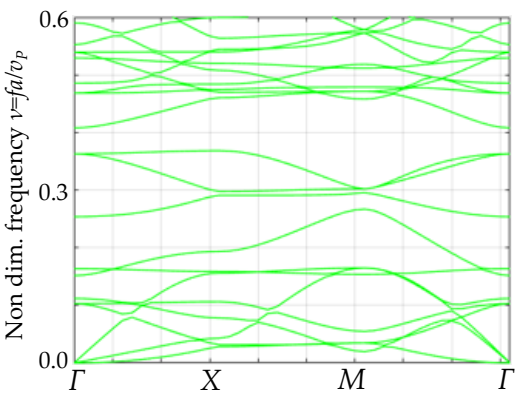

(a) Dispersion relation at $t / a=0.1250$.

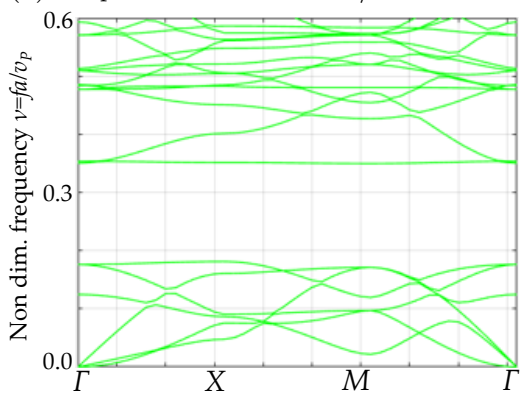

(d) Dispersion relation at $t / a=0.5000$.

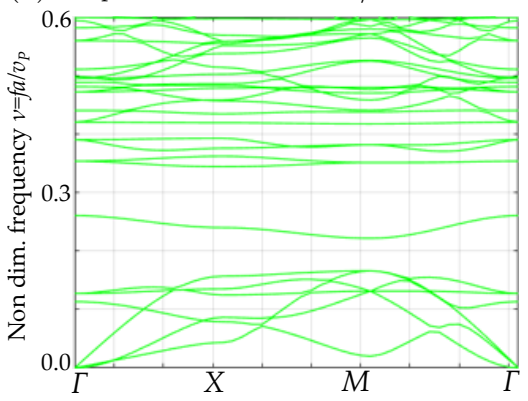

(g) Dispersion relation at $t / a=1.0000$.

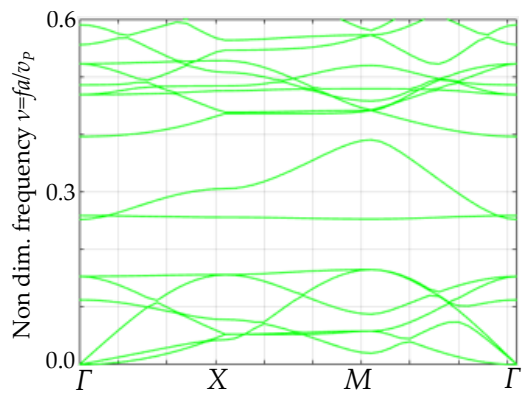

(b) Dispersion relation at $t / a=0.2500$.

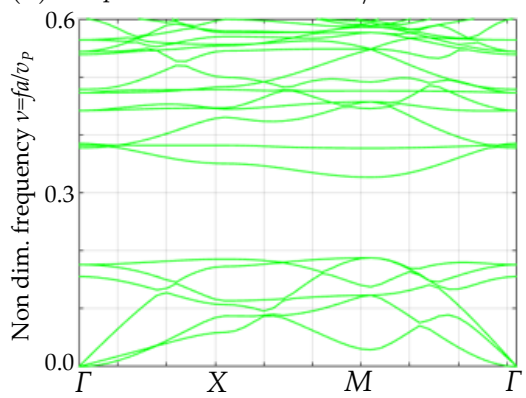

(e) Dispersion relation at $t / a=0.6667$.

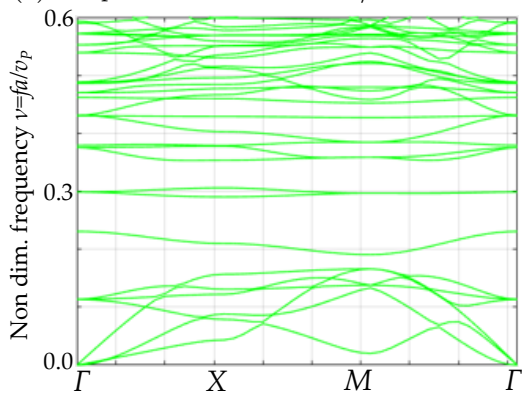

(h) Dispersion relation at $t / a=1.1667$.

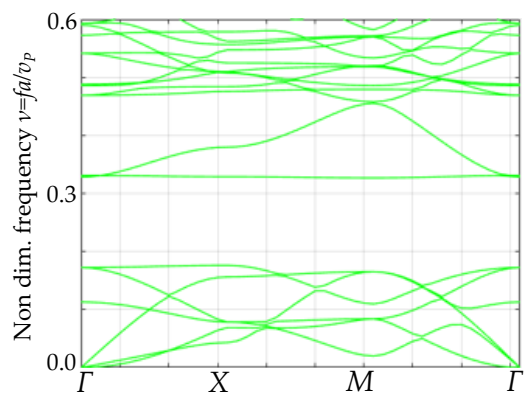

(c) Dispersion relation at $t / a=0.4167$.

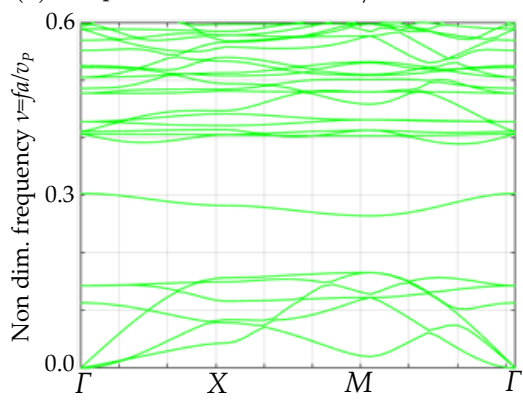

(f) Dispersion relation at $t / a=0.8333$.

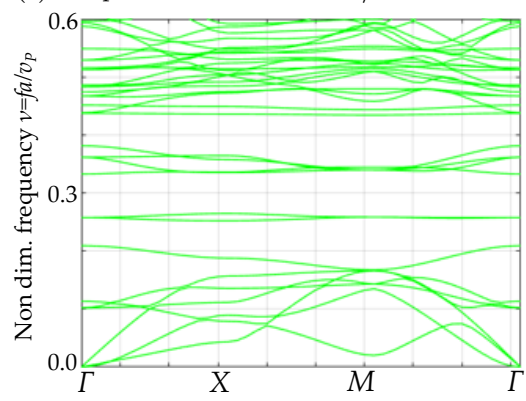

(i) Dispersion relation at $t / a=1.3333$.

Figure 12: Evolution of dispersion relations for different ratios $t / a$. 


\subsection{Typical features of the eigenmodes}

It is interesting to investigate which type of modes are involved in order to understand completely the bandgap variation: Figs. 13, 15 and 17 are used in order to highlight the stresses contents per each mode that define the bandgap at different $t / a$ values. In each of those figures there is one picture for each stress component in which $2 \mathrm{D}$ plane stress and $3 \mathrm{D}$ dispersion relations are superimposed. The 3D dispersion relation is plotted point by point with circles that have the diameter dimension proportional to the relative importance of the specific stress component in that mode. To calculate such a relative importance the sum of the absolute values of each of the six $3 \mathrm{D}$ stress components is calculated all over the mesh, eigenvalue by eigenvalue. From these six sums the percentage of each stress component in the mode is extracted. This is a rough but effective indicator for the problem, because the considered modes are characterized by a global wave shape and there are no local vibrations that conversely would have affected these indicators.

By examining Fig. 13, that is referred to the case $t / a=0.4$, one finds that the $7^{\text {th }}$ mode is characterized mainly by $\tau_{x y}$ stress component, and $8^{\text {th }}$ mode mainly by $\sigma_{x x}$ and $\sigma_{y y}$ stress components. Those two modes are overlooked by the 2D plane stress approximation, since they are dominated by torsional and flexural deformation, respectively. For a better comprehension of this fact, Fig. 14 shows contour plots of the $\tau_{x y}$ stress components for the $7^{\text {th }}$ mode and of the $\sigma_{y y}$ component for the $8^{\text {th }}$ mode in a significant $\mathbf{k - p o i n t : ~ b y ~ c o n s i d e r i n g ~ t h a t ~ o p p o s i t e ~}$ values are obytained on the upper and lower skins, we may infer that some torsional deformation is present in the $7^{\text {th }}$ mode and some bending deformation is present in the $8^{\text {th }}$ mode.

From Fig. 15, that is referred to the case $t / a=0.8333$, it is possile to see that the new (with respect to Fig. 13) $7^{\text {th }}$ mode is characterized mainly by $\tau_{y z}$ and $\tau_{x z}$ stress components, whereas the two modes commented before (Fig. 14) are now at upper frequencies. In Fig. 16, the contour plots of shear stresses for the $7^{\text {th }}$ mode in a significant k-point is plotted. The presence of significant shear stress on the plan orthogonal to the $z$ axis suggests that the mode is dominated by a "drilling" deformation of the disk (i.e. relative rotation of the upper skin with respect to the lower skin, around the $z$ axis). For compatibility reasons, the mode contains also the beam-like torsional deformation of the links between the disks.

For higher $t / a$ ratios, the $3 \mathrm{D}$ effects increase and other $3 \mathrm{D}$ modes (in this case dominated by $\sigma_{z z}$ ) occupy the bandgap (see Fig. 17). 


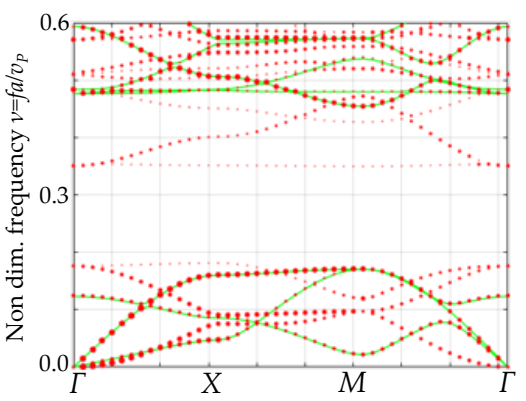

(a) Stress $\sigma_{x x}$.

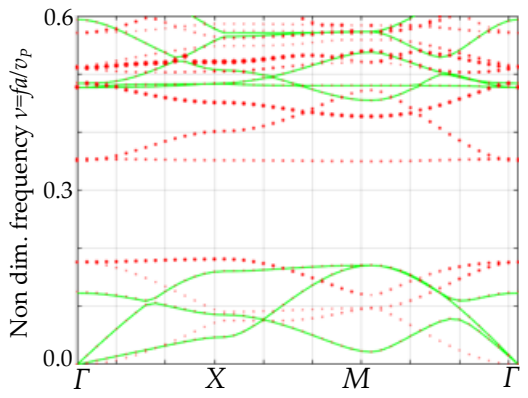

(d) Stress $\tau_{y z}$.

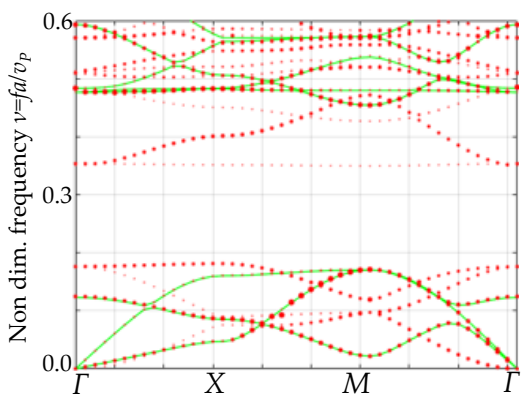

(b) Stress $\sigma_{y y}$

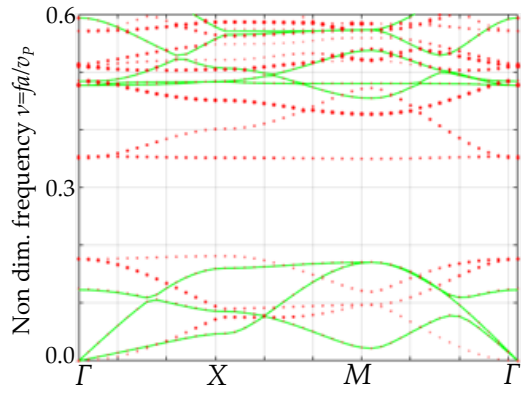

(e) Stress $\tau_{x z}$.

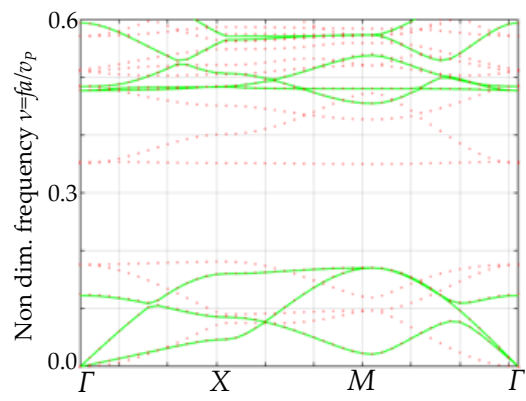

(c) Stress $\sigma_{z z}$.

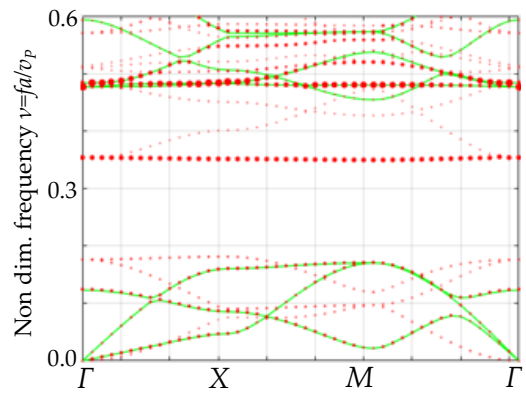

(f) Stress $\tau_{x y}$.

Figure 13: Dispersion relations with comparison between 2D plane stress (green full) and 3D (red dotted) for $t / a=0.5000$. The dot dimension represents the percentage of the stress in the selected mode (each dot must be compared to the others in the same position in the other stresses graphs).

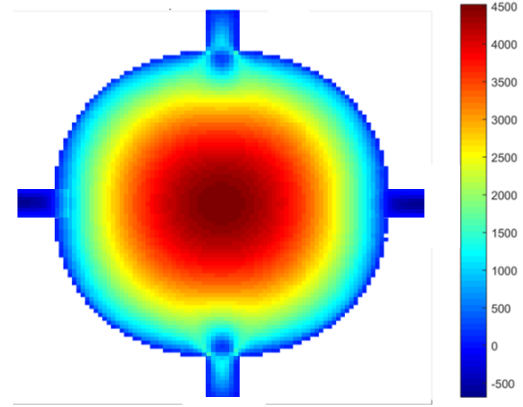

(a) Stress $\tau_{x y}$, th $^{\text {th }}$ eigenvalue, bottom plane.

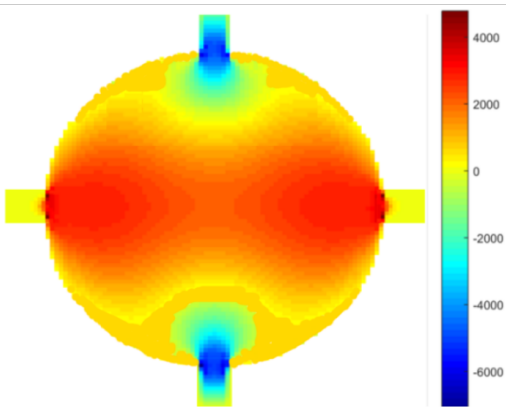

(c) Stress $\sigma_{y y}$, $8^{\text {th }}$ eigenvalue, bottom plane.

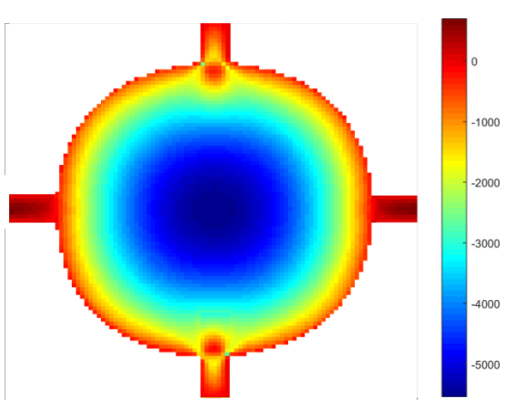

(b) Stress $\tau_{x y}$, 'th eigenvalue, top plane.

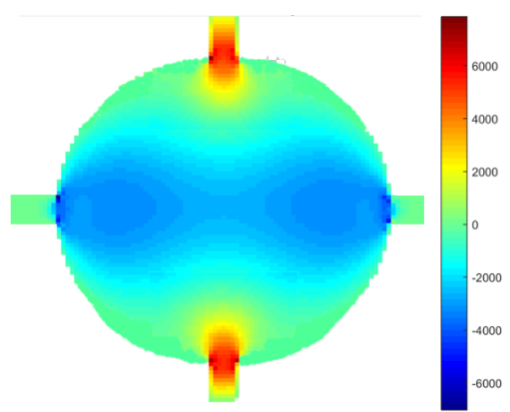

(d) Stress $\sigma_{y y}$, $8^{\text {th }}$ eigenvalue, top plane.

Figure 14: Plot of the stresses for the widest bandgap unit cell with $t / a=0.5000: \tau_{x y}$ for one $\boldsymbol{k}$-point for yth $_{\text {eigenvalue }}$ (bottom and upper plane) and $\sigma_{y y}$ for one $\boldsymbol{k}$-point for $8^{\text {th }}$ eigenvalue (bottom and upper plane).

The $7^{\text {th }}$ contains some torsional strain, the $8^{\text {th }}$ mode contains some bending strain. 


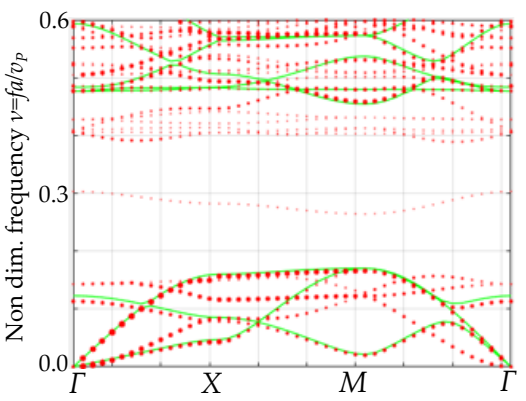

(a) Stress $\sigma_{x x}$.

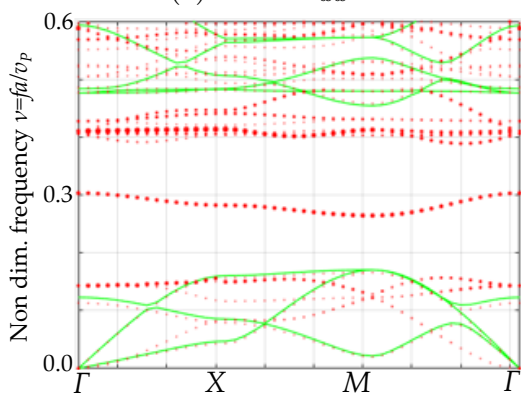

(d) Stress $\tau_{y z}$.

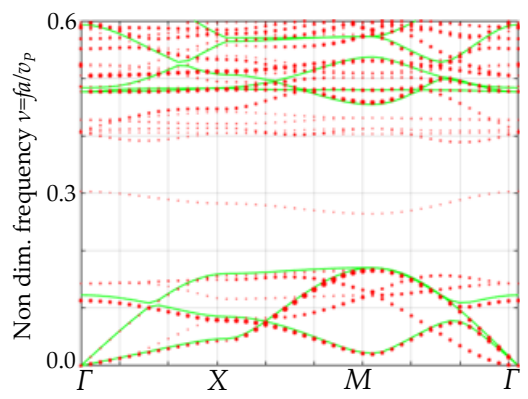

(b) Stress $\sigma_{y y}$.

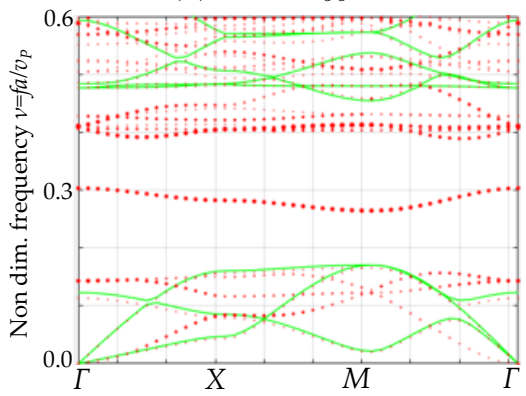

(e) Stress $\tau_{x z}$

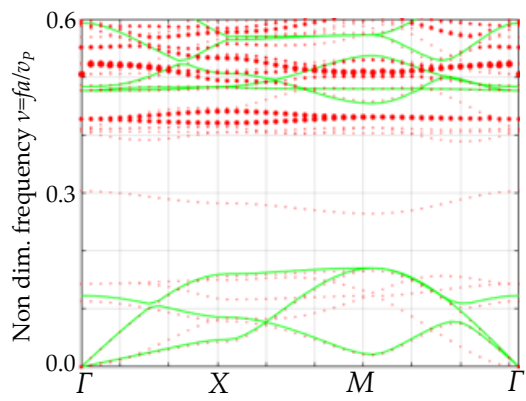

(c) Stress $\sigma_{z z}$.

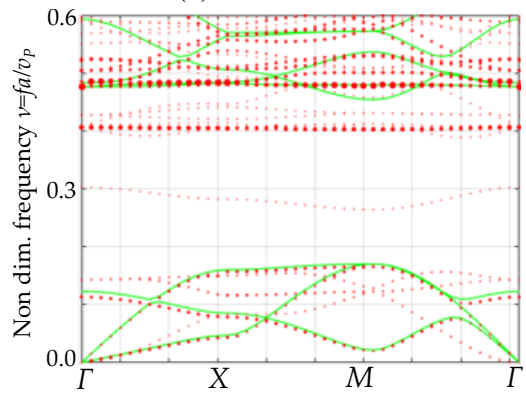

(f) Stress $\tau_{x y}$.

Figure 15: Dispersion relation with comparison between 2D plane stress (green full) and $3 D$ (red dotted) for $t / a=0.8333$. The dot dimension represents the percentage of the stress in the selected mode (each dot must be compared to the others in the same position in the other stresses graphs).

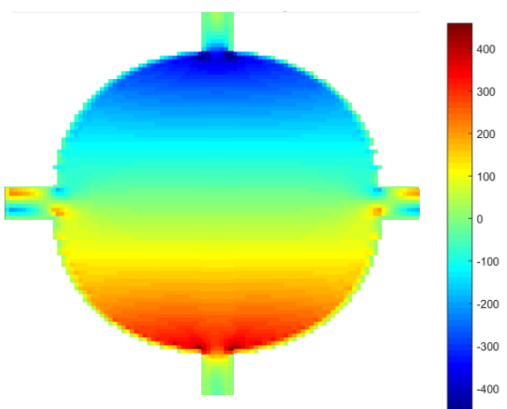

(a) Stress $\tau_{x z}$, rth eigenvalue, bottom plane.

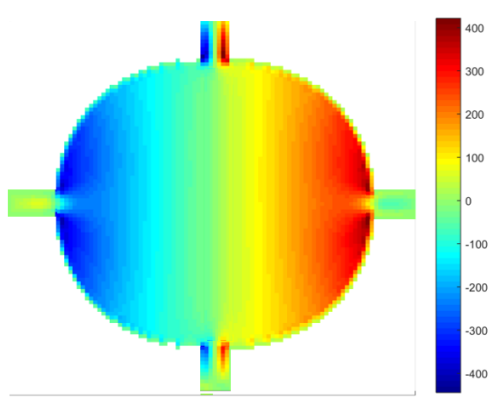

(d) Stress $\tau_{y z}$, rth eigenvalue, bottom plane.

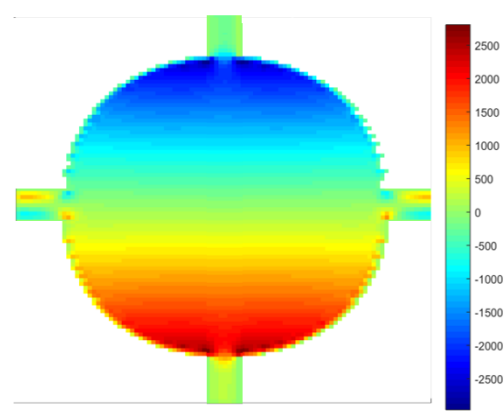

(b) Stress $\tau_{x z}$, $7^{\text {th }}$ eigenvalue, middle plane.

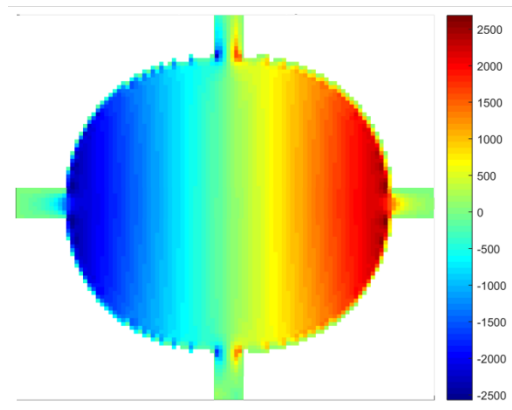

(e) Stress $\tau_{y z}$, rth eigenvalue, middle plane.

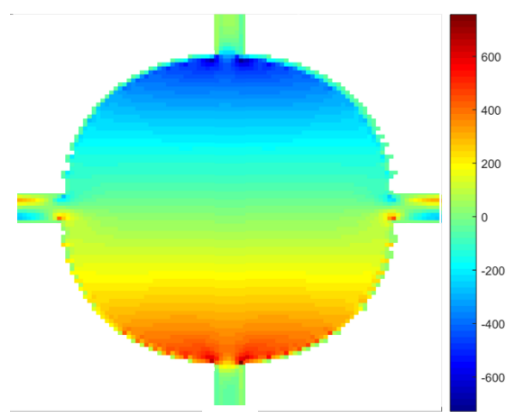

(c) Stress $\tau_{x z}$, th $^{\text {th }}$ eigenvalue, top plane.

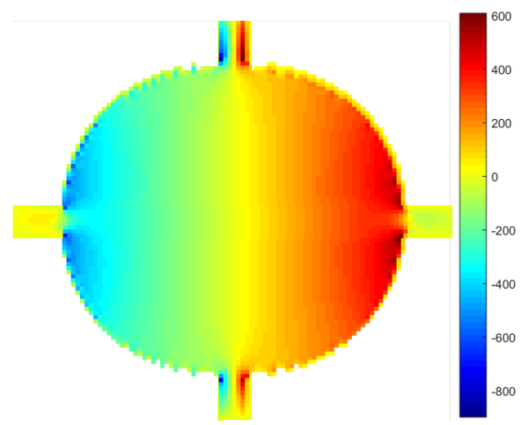

(f) Stress $\tau_{y z}$, rth eigenvalue, top plane.

Figure 16: Plot of the stresses for the widest bandgap unit cell with $t / a=0.8333: \tau_{x z}$ for one $\boldsymbol{k}$-point for th $^{\text {th }}$ eigenvalue (bottom, middle and upper plane) and $\tau_{y z}$ for one $\boldsymbol{k}$-point for the same eigenvalue (bottom, middle and upper plane).

The rth $^{\text {th }}$ mode is characterized by the drilling deformation of the central disk. 


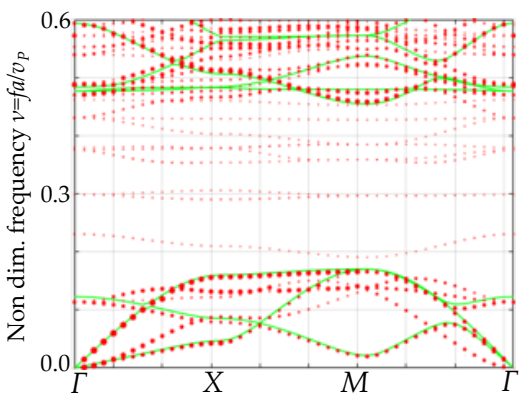

(a) Stress $\sigma_{x x}$.

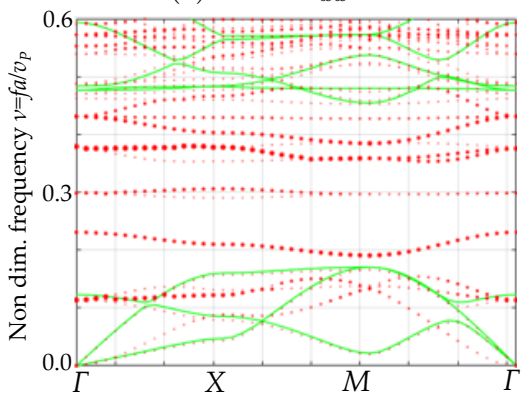

(d) Stress $\tau_{y z}$.

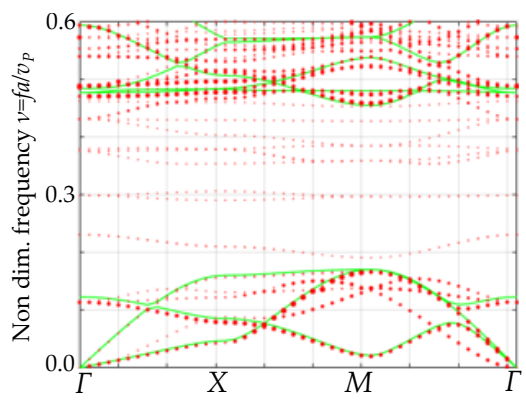

(b) Stress $\sigma_{y y}$.

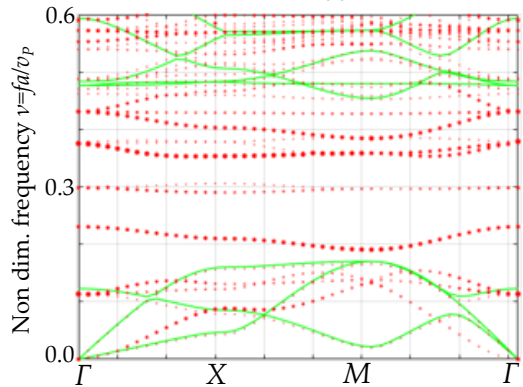

(e) Stress $\tau_{x z}$

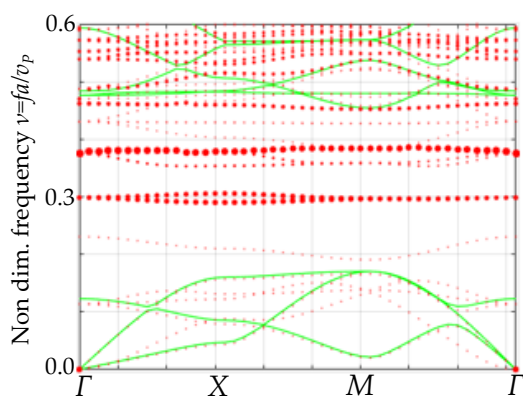

(c) Stress $\sigma_{z z}$.

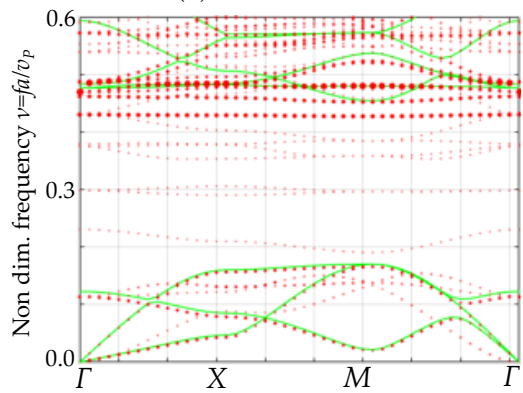

(f) Stress $\tau_{x y}$.

Figure 17: Dispersion relation with comparison between 2D plane stress (green full) and 3D (red dotted) for t/a $=1.1667$. The dot dimension represents the percentage of the stress in the selected mode (each dot must be compared to the others in the same position in the other stresses graphs).

\section{Comparative analysis of the achieved results}

\subsection{Analysis with different materials typical of MEMS industry}

In the previous Sections we considered a specific material in our computations, namely GaN, in view of the possible application of optimal phononic crystals in MEMS unreleased resonators [10]. For comparative purposes, it is now interesting to see how the optimal topology found in the previous Sections works for different materials of common use in MEMS industry, i.e. aluminium nitride (AlN) and epitaxial silicon ( $\mathrm{Si}$ ), whose mechanical properties are summarizaed in Table 1. Reference is made to the optimal shape of the first useful bandgap, between the $6^{\text {th }}$ and $7^{\text {th }}$ eigenmodes: Fig. 18 describes the achieved results for the three materials, in the case of thickness-to-width ratio equal to 0.500 .

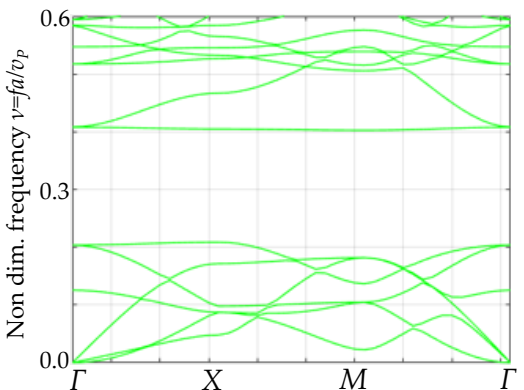

(a) $A l N$.

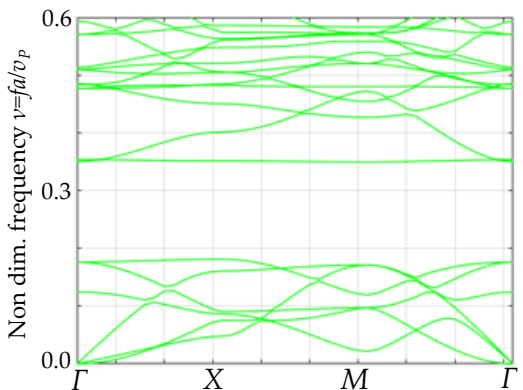

(b) GaN.

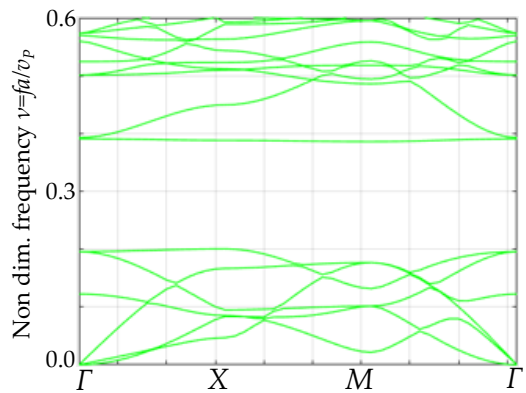

(c) Si.

Figure 18: Comparison between dispersion relations of different materials used in MEMS industry for $t / a=0.500$.

By examining the achieved results, one can infer that the dispersion relation for solid-air PnC depends strongly on the geometry of the hole, and less on the material properties: the frequencies content in the PnCs is similar altough the ratios of the materials constants in the three materials of Fig. 18 are not so close (see Tab. 1); the relative bandgap for the same optimal shape is $69 \%$ for AlN, $64 \%$ for GaN and $67 \%$ for $\mathrm{Si}$. 


\subsection{Comparison with results in literature}

A large number of interesting results have been presented in very recent works: [20], [21] and [23] perform optimization by means of the genetic algorithm, [26] by means of a different BESO approach. All of them use Si as reference material (see Tab. 1) and could be compared to the result of Fig. 18 (c), endowed with the gap to mid-gap ratio for full frequencies bandgap of $67 \%$.

First, we consider the results presented in [23], that is the only paper where the optimization procedure is applied to fully 3D problems. In order to perform a fair comparison, full bandgap topologies (for both symmetric and anti-symmetric modes) are considered from the previous work for ratio $t / a=0.500$. The topologies indicated in that paper as TH-1 to TH-10 are the optimal ones and they span gap to mid-gap ratio between $85 \%$ and $20 \%$, depending on the position along the Pareto frontier. The results of the present work can be compared to TH-3, which has a similar in-plane stiffness with respect to our optimal topology. Therefore, the result to compare with is around $60 \%$ for gap to mid-gap percentage, which shows that our algorithm performs slightly better. The maximum relative bandgap presented in [23] is equal to $85 \%$ and corresponds to the first point on the Pareto frontier, associated with the lowest relative elastic modulus: we can reach that value if we relax our constraint: for example, if we consider that the border constraint is constituted of a single element line our optimal relative bandgap jumps to $100 \%$, see Fig. 19.

As a result of the comparison, we can conclude that the present approach finds equivalent or slightly better topologies in terms of gap to mid-gap ratios, but extremely different on terms of feasibility in microfabrication due to the contractible hole topology. Furthermore, as already stressed before, the optimization procedure is faster and does not take into account air modelling.

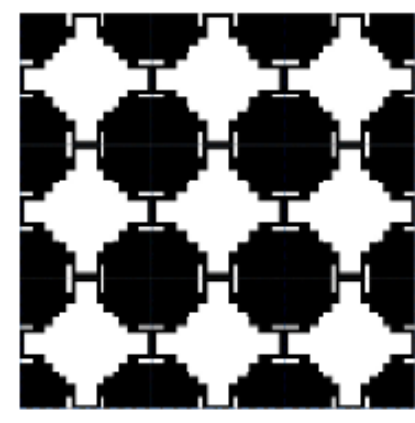

(a)

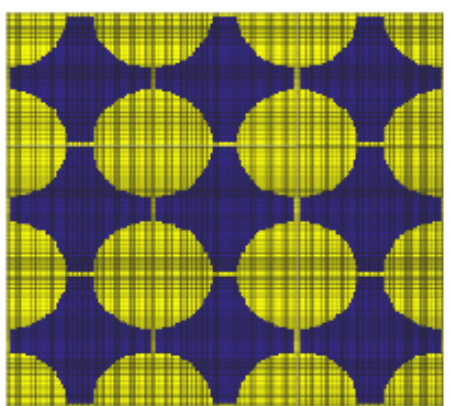

(b)

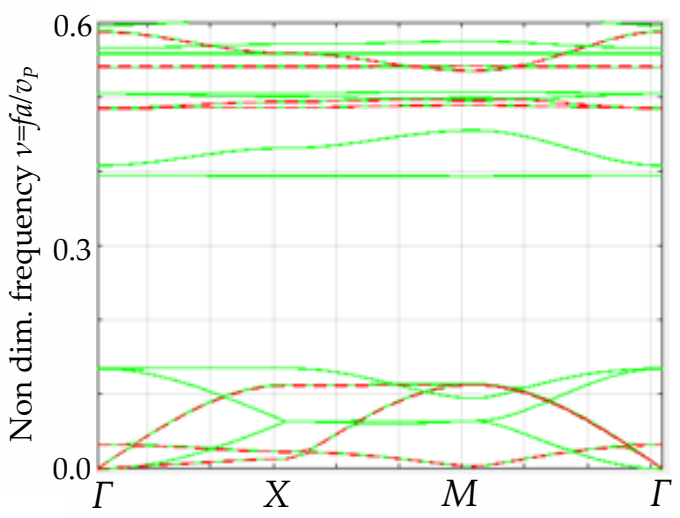

(c)

Figure 19: Comparison with previous work: (a) optimal cell for the case TH-1 in [23]; (b) optimal cell obtained with the present approach, with border constraint of 1 element; (c) dispersion diagram for the cell (b), 3D modes in green solid line, $2 D$ plane stress modes in red dashed line.

The Authors in [20], [21] and [26] perform the optimization on 2D PnCs with plane stress/plane strain behaviour and therefore they do not take into account torsional and bending modes.

The optimal topology presented in [20] for both in-plane and out-of-plane waves is endowed with $63 \%$ of gap to mid-gap bandgap percentage, which is less than the $67 \%$ found in this work (see Fig. 18 (c)) with 3-line constraint and far from the $100 \%$ with 1-line constraint (see Fig. 19). It is worth reminding that the out-of-plane modes considered in [20] are a small subset of the complete 3D modes, that are considered in the present approach. The other papers, [21] and [26], present gap to mid-gap ratios slightly above $100 \%$ which is comparable to our full 3D $100 \%$. As said before this is mainly due to the fact that they do not take into account the torsional and flexural modes. If we consider the in-plane modes only, we find that the relative width of the first bandgap attains $120 \%$ (see red dotted lines in Fig. 19c); the analogous maximum value achieved in [21] is $121.6 \%$ and in [26] it is $108.32 \%$. It is worth noting that their topologies present several holes within the unit cell, which, as for [23], is not sound for microfabrication and therefore not the goal of the present work. 


\section{Conclusions}

The present paper represents a contribution to the optimal design of PnCs with reference to their practical application in MEMS resonators. The problem of shape optimization for achieving the maximum relative width of bandgap is tackled by means of an original algorithm, which has been developed in the framework of BESO technique. The optimization procedure is applied to the case of 3D modes for the wave propagation inside PnC slabs, whereas most of the previous works are focussed on $2 \mathrm{D}$ approximations. The algorithm has been specifically tuned in order to achieve holes in the form of contractible space, so that the final design is fully compatible with the typical features of microfabrication. A suitable constraint has been introduced in order to guarantee the structural worthiness of the optimal PnC. The algorithm is very fast, if compared to other proposals in the literature, and has been applied sistematically for different eigenvalues. The typical effects of 3D behaviour have been carefully considered, with reference to the variation of the aspect ratio of the unit cell.

The performance of the algorithm has been compared with the literature on the same topic. In spite of the aforementioned constraints, the optimal shapes obtained in the present paper compare very well to the previous proposals: the relative bandgap widths obtained here are better than the values achieved in the only paper which treats fully 3D problems. Similarly, a positive comparison is obtained with respect to previous works which adopt 2D approximations. The most important feature of our optimal $\mathrm{PnC}$ is that they can be directly implemented in MEMS resonators, with great advantages with respect to previous proposals. For instance, a similar shape is used in [39] for a layered material (AlN with top and bottom Pt layers). In that paper, the relative bandgap width is equal to $27 \%$. We replaced that shape with our optimal shape, with the same material and the same dimensions of the unit cell: our relative bandgap is equal to $53 \%$, which is by far better than the original proposal. The Authors in [40] considered a different $\mathrm{PnC}$ with square shape of the solid portions, instead of our circular shape: the relative bandgap in [40] is $30 \%$, while our optimal shape reaches $50 \%$ for that material and that dimension. We can therefore conclude that the application of the proposed algorithm may lead to a substantial improvement of the performance of PnC in MEMS resonators.

\section{Acknowledgments}

The authors wants to aknowledge Rocca Foundation for the opportunity given to develop this work. Author L. D'A. acknowledges the contribution of MIUR: Project PRIN15-2015LYYXA8 "Multi-scale mechanical models for the design and optimization of microstructured smart materials and metamaterials". The Authors L.D'A. and R.A. wish to thank Dr. Matteo Bruggi for the stimulating discussions and the precious help in the early stage of the development of the optimization algorithm.

\section{References}

[1] T. Gorishnyy, M. Maldovan, C. Ullal, E. Thomas, Sound ideas, Physics World 18 (12) (2005) 24-29.

[2] M. Maldovan, Sound and heat revolutions in phononics, Nature 503 (7475) (2013) 209-217.

[3] T. Delpero, S. Schoenwald, A. Zemp, A. Bergamini, Structural engineering of three-dimensional phononic crystals, Journal of Sound and Vibration 363 (2015) 156-165.

[4] H. Zhu, F. Semperlotti, Metamaterial based embedded acoustic filters for structural applications, AIP Advances 3 (9) (2013) 092121.

[5] Z. Chen, B. Guo, Y. Yang, C. Cheng, Metamaterials-based enhanced energy harvesting: A review, Physica B: Condensed Matter 438 (2014) 1-8.

[6] M. Gei, A. Movchan, D. Bigoni, Band-gap shift and defect-induced annihilation in prestressed elastic structures, Journal of Applied Physics 105 (6) (2009) 063507.

[7] M. Gei, D. Bigoni, A. B. Movchan, M. Bacca, Band-gap properties of prestressed structures, in: R. V. Craster, S. Guenneau (Eds.), Acoustic Metamaterials: Negative Refraction, Imaging, Lensing and Cloaking, Springer Netherlands, 2013, pp. 61-82.

[8] P. Wang, J. Shim, K. Bertoldi, Effects of geometric and material nonlinearities on tunable band gaps and lowfrequency directionality of phononic crystals, Physical Review B - Condensed Matter and Materials Physics 88 (1) (2013) 014304. 
[9] J. Segovia-Fernandez, M. Cremonesi, C. Cassella, A. Frangi, G. Piazza, Anchor losses in aln contour mode resonators, Journal of Microelectromechanical Systems 24 (2) (2015) 265-275.

[10] S. Wang, L. C. Popa, D. Weinstein, GaN MEMS resonator using a folded phononic crystal structure, in: Proc. of Hilton Head Workshop 2014: A Solid-State Sensors, Actuators and Microsystems Workshop, 2014, pp. $72-75$.

[11] B. Bahr, R. Marathe, D. Weinstein, Phononic crystals for acoustic confinement in CMOS-MEMS resonators, in: Proc. of IFCS 2014 - 2014 IEEE International Frequency Control Symposium, 2014, p. 6859980.

[12] T.-T. Wu, J.-C. Hsu, J.-H. Sun, Phononic plate waves, IEEE Transactions on Ultrasonics, Ferroelectrics, and Frequency Control 58 (10) (2011) 2146-2161.

[13] L. D'Alessandro, E. Belloni, R. Ardito, A. Corigliano, F. Braghin, Modeling and experimental verification of an ultra-wide bandgap in 3D phononic crystal, Applied Physics Letters 109 (22) (2016) 221907.

[14] L. Montemayor, V. Chernow, J. Greer, Materials by design: Using architecture in material design to reach new property spaces, MRS Bulletin 40 (12) (2015) 1122-1129.

[15] A. Kruisová, H. Seiner, P. Sedlák, M. Landa, B. Román-Manso, P. Miranzo, M. Belmonte, Acoustic metamaterial behavior of three-dimensional periodic architectures assembled by robocasting, Applied Physics Letters 105 (21) (2014) 211904.

[16] O. Sigmund, J. Jensen, Systematic design of phononic band-gap materials and structures by topology optimization, Philosophical Transactions of the Royal Society A: Mathematical, Physical and Engineering Sciences 361 (1806) (2003) 1001-1019.

[17] G. Gazonas, D. Weile, R. Wildman, A. Mohan, Genetic algorithm optimization of phononic bandgap structures, International Journal of Solids and Structures 43 (18-19) (2006) 5851-5866.

[18] O. Bilal, M. Hussein, Optimization of phononic crystals for the simultaneous attenuation of out-of-plane and in-plane waves, in: ASME 2011 International Mechanical Engineering Congress and Exposition, IMECE 2011, Vol. 8, 2011, pp. 969-972.

[19] H.-W. Dong, X.-X. Su, Y.-S. Wang, C. Zhang, Topology optimization of two-dimensional asymmetrical phononic crystals, Physics Letters, Section A: General, Atomic and Solid State Physics 378 (4) (2014) 434441.

[20] O. Bilal, M. Hussein, Ultrawide phononic band gap for combined in-plane and out-of-plane waves, Physical Review E - Statistical, Nonlinear, and Soft Matter Physics 84 (6) (2011) 065701.

[21] H.-W. Dong, X.-X. Su, Y.-S. Wang, Multi-objective optimization of two-dimensional porous phononic crystals, Journal of Physics D: Applied Physics 47 (15) (2014) 155301.

[22] H.-W. Dong, Y.-S. Wang, Y.-F. Wang, C. Zhang, Reducing symmetry in topology optimization of twodimensional porous phononic crystals, AIP Advances 5 (11) (2015) 117149.

[23] S. Hedayatrasa, K. Abhary, M. Uddin, C.-T. Ng, Optimum design of phononic crystal perforated plate structures for widest bandgap of fundamental guided wave modes and maximized in-plane stiffness, Journal of the Mechanics and Physics of Solids 89 (2016) 31-58.

[24] F. Meng, X. Huang, B. Jia, Bi-directional evolutionary optimization for photonic band gap structures, Journal of Computational Physics 302 (2015) 393-404.

[25] Y. Li, X. Huang, F. Meng, S. Zhou, Evolutionary topological design for phononic band gap crystals, Structural and Multidisciplinary Optimization 54 (3) (2016) 595-617.

[26] Y. Li, X. Huang, S. Zhou, Topological design of cellular phononic band gap crystals, Materials 9 (3) (2016) 186.

[27] M. Hussein, Reduced Bloch mode expansion for periodic media band structure calculations, Proceedings of the Royal Society A: Mathematical, Physical and Engineering Sciences 465 (2109) (2009) 2825-2848. 
[28] M. Collet, M. Ouisse, M. Ruzzene, M. Ichchou, Floquet-Bloch decomposition for the computation of dispersion of two-dimensional periodic, damped mechanical systems, International Journal of Solids and Structures 48 (20) (2011) 2837-2848.

[29] C. Kittel, Introduction to Solid State Physics, 8th Edition, John Wiley \& Sons, 2004.

[30] J.-H. Sun, T.-T. Wu, Propagation of acoustic waves in phononic-crystal plates and waveguides using a finitedifference time-domain method, Physical Review B - Condensed Matter and Materials Physics 76 (10) (2007) 104304.

[31] L. D'Alessandro, B. Bahr, L. Daniel, D. Weinstein, R. Ardito, BESO approach to topology optimization of GaN phononic crystals, in: ECCOMAS Congress 2016 - Proceedings of the 7th European Congress on Computational Methods in Applied Sciences and Engineering, Vol. 2, 2016, pp. 3583-3595.

[32] A. Srikantha Phani, J. Woodhouse, N. Fleck, Wave propagation in two-dimensional periodic lattices, Journal of the Acoustical Society of America 119 (4) (2006) 1995-2005.

[33] L. Brillouin, Wave Propagation in Periodic Structures, 2nd Edition, Dover Publication, 1953.

[34] Q. Li, G. Steven, Y. Xie, A simple checkerboard suppression algorithm for evolutionary structural optimization, Structural and Multidisciplinary Optimization 22 (3) (2001) 230-239.

[35] X. Yang, Y. Xie, J. Liu, G. Parks, P. Clarkson, Perimeter control in the bidirectional evolutionary optimization method, Structural and Multidisciplinary Optimization 24 (6) (2003) 430-440.

[36] Y. Xie, G. Steven, Evolutionary Structural Optimization, Springer Verlag, 1997.

[37] R. Grandhi, Structural optimization with frequency constraints-a review, AIAA Journal 31 (12) (1993) 22962303.

[38] J. Han, E. Rudnyi, J. Korvink, Efficient optimization of transient dynamic problems in MEMS devices using model order reduction, Journal of Micromechanics and Microengineering 15 (4) (2005) 822-832.

[39] N.-K. Kuo, C. Zuo, G. Piazza, Microscale inverse acoustic band gap structure in aluminum nitride, Applied Physics Letters 95 (9) (2009) 093501.

[40] T. Alegre, A. Safavi-Naeini, M. Winger, O. Painter, Quasi-two-dimensional optomechanical crystals with a complete phononic bandgap, Optics Express 19 (6) (2011) 5658-5669. 